Federal Reserve Bank of Minneapolis

Research Department Staff Report 222

Revised January 1998

\title{
Individual and Aggregate Real Balances in a Random-Matching Model
}

\author{
Ruilin Zhou* \\ Federal Reserve Bank of Minneapolis \\ and University of Pennsylvania
}

\begin{abstract}
This paper investigates the characteristics of stationary single-price equilibrium in a monetary randommatching model where agents can hold an arbitrary amount of divisible money and where production is costly. At such an equilibrium, agents' money holdings are endogenously determined and uniformly bounded. A refinement of weakly undominated strategies is argued to be necessary. It is shown that a continuum of single-price equilibria indexed by the aggregate real-money balance exists if one such equilibrium exists. Equilibria with different money-holdings upper bounds, hence different distributions, but with identical aggregate real-money balances, can coexist.
\end{abstract}

*I thank Harold Cole, Dean Corbae, Ed Green, Neil Wallace, and Randall Wright for helpful conversations. The views expressed herein are those of the author and not necessarily those of the Federal Reserve Bank of Minneapolis or the Federal Reserve System. 


\section{INTRODUCTION}

How is price formed in a decentralized trading environment? How do individual traders make their pricing decision based on their money balances? How does the aggregate real-money balance manifest itself in its effect on economywide trades? There are many such questions one hopes to answer with the help of search-theoretic models of money that endogenize monetary trade as a means of overcoming market frictions. Two key simplifying assumptions in the literature limit the ability of the models to answer these questions completely: indivisibility of traded objects (including both money and goods) and a unit upper bound on holdings of objects. Together, these assumptions imply that the only transaction pattern is one-for-one swapping of goods and/or money. Shi [9] and Trejos and Wright [10] keep both constraints on money but provide scope for price flexibility by endowing each agent with the capacity to produce a divisible perishable good at a cost that varies with the amount produced. I study a model that is dual to theirs in the sense that the indivisibility of production is maintained, but money is divisible and agents can hold any amount of it. ${ }^{1}$

Green and Zhou [3] (GZ hereafter) study a special case of the model that I study in this paper in which an agent obtains his one unit of production good costlessly. The main result of GZ is the existence of a continuum of stationary single-price equilibria which are indexed by the aggregate real-money balance and are welfare-ranked. The greater is the real-money balance, the higher is welfare. However, a gift-giving, nonmonetary equilibrium always exists and Pareto dominates any monetary equilibrium.

Unfortunately, the costless-production assumption has some unappealing consequences. The existence of a gift-giving, nonmonetary equilibrium, or equivalently, the inessentiality of money in facilitating exchange, is a direct consequence of the assumption. Questions also arise as to whether the GZ results concerning the existence of a continuum of equilibria and their ranking in terms of welfare as an increasing function of the aggregate real money balances are robust with respect to changes of this assumption. If goods can be obtained instantaneously without a cost, then there seems to be no "real" aspect of the economy that pins down the value of money.

To answer these questions, I study stationary single-price equilibrium in a costly-production environment. ${ }^{2}$ The presence of a production cost raises a subtle conceptual problem in defining the single-price equilibrium. The production cost induces an endogenously determined upper

\footnotetext{
${ }^{1}$ Some parallel work has been done. Camera and Corbae [1], Molico [6], and Wallace [11] endow agents with the ability to produce divisible perishable goods and adopt a full-information bargaining mechanism in pairwise trading. Camera and Corbae [1] and Wallace [11] allow agents to hold multiple units of money up to a finite upper bound. Molico [6] computationally studies a model without an upper bound on money holdings. A recent paper by Rocheteau [8] studies a similar model as mine with indivisible money.

${ }^{2} \mathrm{I}$ am indebted to Dean Corbae for correcting my initial erroneous conjecture that stationary distribution of a single-price equilibrium would not exist in a costly-production environment.
} 
bound on agents' money holdings at which the cost of production exceeds agents' marginal value of holding money. That is, "rich" agents stop selling and accumulating money at the prevailing price, and higher offers will not be accepted in equilibrium. Hence, the equilibrium does not have full support. This is not a problem in studies with an exogenous upper bound on holdings of money where the equilibrium support is fixed by the bound or in GZ's costless-production environment in which the equilibrium support is always infinite. I adopt a weak form of equilibrium refinement - elimination of weakly dominated strategies - to deal with the partial-support problem. The equilibrium concept is Bayesian Nash equilibrium with weakly undominated strategies.

The paper develops a set of sufficient conditions under which a single-price equilibrium exists. Applying these conditions, I study some special cases of the single-price equilibrium. The analysis shows that, generically, if one single-price equilibrium exists, then a continuum of similar equilibria indexed by price or aggregate real-money balance also exists. Hence, a main result of GZ survives the introduction of costly production. The presence of the production cost also eliminates the gift-giving, nonmonetary equilibrium.

The study of a particular single-price equilibrium that resembles equilibria in models with an exogenous upper bound on holdings of indivisible money demonstrates the different effect of an endogenous versus exogenous money-holdings upper bound on the existence of the equilibrium. For a given single-price equilibrium, had the endogenous upper bound on holdings of money been replaced by the same exogenous upper bound, the equilibrium existence region will be larger. That is, the endogeneity imposes additional restrictions on the existence of an equilibrium.

The single-price equilibrium in this paper is qualitatively very different from that of the costless-production environment of GZ. Besides the difference in equilibrium support, the steadystate welfare ranking of single-price equilibria in this model is, in general, not monotonic in aggregate real-money balances. The measure of agents whose money holdings reach the upper bound and who, therefore, choose not to be productive until they have first spent some money affects the welfare negatively since the existence of this group of people slows down trades.

In addition to the continuum of equilibria as in GZ, the model possesses another dimension of multiplicity. For a given real-money balance, single-price equilibria with distinct upper bounds on money holdings can coexist. They differ in the distribution of money holdings, velocity of trading, and, therefore, welfare. This result shows that the aggregate real-money balance is not the only endogenous factor that characterizes equilibrium allocations and that the distribution of money holdings is another important factor which may not be uniquely determined by the aggregate real-money balance.

\section{THE MODEL}

There is a continuum of infinitely lived agents with a mass of measure 1. The population is 
equally divided into $k$ types, $k \geq 3$. Time is continuous. There are $k+1$ goods. Of these goods, $k$ (indexed by 1 through $k$ ) are indivisible, immediately perishable goods that are produced by the agents. The remaining good is a divisible, perfectly durable, fiat-money object with a constant total nominal stock $M$. A type $i$ agent can produce one unit of good $i+1(\bmod k)$ at a utility $\operatorname{cost} c>0$ at any time. He consumes only good $i$, from which he derives an instantaneous utility $u$. An agent can hold any amount of money. Each agent maximizes discounted expected utility with discount rate $\gamma$.

Agents meet pairwise randomly according to a Poisson process with parameter $\mu$. The specialization in preference and production ensures the lack of a double coincidence of wants between any pair of agents. Consumption goods cannot be used as commodity money because they are perishable. Thus, trade must involve fiat money as a medium of exchange. An agent is characterized by his type and the amount of money he holds. Agents' types are publicly known, but their money holdings and trading histories are private information. Pairwise trading is conducted through a seller-posting-price mechanism. When a type $i$ agent who has fiat money (buyer) meets a type $i-1$ agent who can produce his desired good (seller), the seller posts an offer at which he is willing to sell, and the buyer must either accept or reject it. Trade occurs if and only if the offer is accepted, and, in that case, the buyer pays exactly the seller's offer price.

I will focus on stationary symmetric equilibrium where all agents with identical money holdings act alike and agents' trading strategy is time-invariant.

Define $\mathbb{R}_{+}^{*}=\mathbb{R}_{+} \cup\{+\infty\}$. Let $H$ denote the stationary distribution of money holdings in the economy, which is a measure on $\mathbb{R}_{+}$. A generic type $i$ agent's trading strategy consists of a pair of functions: an offer strategy $\omega: \mathbb{R}_{+} \rightarrow \mathbb{R}_{+}^{*}$, which specifies the offer that an agent makes as a seller when he meets a type $i+1$ agent, and a reservation-price strategy $\rho: \mathbb{R}_{+} \rightarrow \mathbb{R}_{+}$, which specifies the maximum willingness to pay as a buyer when he meets a type $i-1$ agent. A symmetric strategy profile and a stationary distribution of money holdings imply stationary distributions of offers, called $\Omega$,

$$
\forall x \in \mathbb{R}_{+}^{*} \quad \Omega(x)=H\{\eta \mid \omega(\eta) \leq x\}
$$

and of reservation prices, called $R$,

$$
\forall x \in \mathbb{R}_{+} \quad R(x)=H\{\eta \mid \rho(\eta)<x\}
$$

Note that, for convenience, $R$ is defined to be continuous from the left, rather than from the right as is conventional.

Let $\mathcal{V}: \mathbb{R}_{+} \rightarrow \mathbb{R}_{+}$denote the value function which specifies the maximum attainable expected discounted utility of an agent as a function of his current money holdings. Since agents are free to dispose of money, the value function is nondecreasing. 
LEMma 1. The value function $\mathcal{V}$ is weakly increasing in money holdings.

A trade meeting between a buyer with money holdings $\eta_{b}$ and a seller with money holdings $\eta_{s}$ entails an extensive-form bargaining game. This is actually a Bayesian game, since the two traders' money holdings are not observable to one another. Given the reservation-price distribution $R$, the seller posts an offer $o$ to maximize his expected discounted net gain from the trade, weighing the potential gain of an increase of money holdings to $\eta_{s}+o$ and the utility cost of production c. Note that, given the positive production cost, it is never optimal to give away one's good for free. The buyer then checks the offer $o$ against his reservation price $\rho\left(\eta_{b}\right)$. He accepts the offer if it does not exceed $\rho\left(\eta_{b}\right)$. The reservation price $\rho\left(\eta_{b}\right)$ must satisfy the feasibility constraint:

$$
\rho\left(\eta_{b}\right) \leq \eta_{b}
$$

As it will become clear later, the equilibria I study in this paper do not have full support. Issues regarding off-equilibrium path actions arise that may have implications for equilibrium. For buyers, there may be out-off-equilibrium offers to consider. Sellers need to evaluate different potential offers, some of which, if accepted, will increase money holdings to a level that is outside the support. In such a case, sellers have to consider offers which the probability of acceptance is zero. The particular offer sellers chose to make among the set of zero-probability-acceptance offers will affect the equilibrium outcome. To deal with these issues systematically, some form of equilibrium refinement is needed. I will adopt a weak form of refinement - eliminating weakly dominated strategies within a trade meeting.

For the trade meeting between a buyer with money holdings $\eta_{b}$ and a seller with money holdings $\eta_{s}$, suppose that the seller's offer is $o$ and the buyer's reservation price is $r$. Given the trading mechanism, let $\Pi_{s}$ denote the net gain for the seller,

$$
\Pi_{s}\left(\eta_{s}, o, r\right)= \begin{cases}\mathcal{V}\left(\eta_{s}+o\right)-c-\mathcal{V}\left(\eta_{s}\right) & \text { if } o \leq r \\ 0 & \text { otherwise }\end{cases}
$$

and let $\Pi_{b}$ denote the net gain for the buyer,

$$
\Pi_{b}\left(\eta_{b}, o, r\right)= \begin{cases}u+\mathcal{V}\left(\eta_{b}-o\right)-\mathcal{V}\left(\eta_{b}\right) & \text { if } o \leq r \\ 0 & \text { otherwise. }\end{cases}
$$

Then, offer $o$ weakly dominates $o^{\prime}$ if

$$
\begin{array}{ll}
\forall r \in \mathbb{R}_{+} & \Pi_{s}\left(\eta_{s}, o, r\right) \geq \Pi_{s}\left(\eta_{s}, o^{\prime}, r\right) \\
\exists \hat{r} \in \mathbb{R}_{+} & \Pi_{s}\left(\eta_{s}, o, \hat{r}\right)>\Pi_{s}\left(\eta_{s}, o^{\prime}, \hat{r}\right)
\end{array}
$$


and reservation price $r$ weakly dominates $r^{\prime}$ if

$$
\begin{array}{ll}
\forall o \in \mathbb{R}_{+}^{*} & \Pi_{b}\left(\eta_{b}, o, r\right) \geq \Pi_{b}\left(\eta_{b}, o, r^{\prime}\right) \\
\exists \hat{o} \in \mathbb{R}_{+}^{*} & \Pi_{b}\left(\eta_{b}, \hat{o}, r\right)>\Pi_{b}\left(\eta_{b}, \hat{o}, r^{\prime}\right) .
\end{array}
$$

Offer $o$ and reservation price $r$ are weakly undominated if they are not weakly dominated by any other offer $o^{\prime}$ and reservation price $r^{\prime}$, respectively. Given this definition, and given that the value function is weakly increasing, weakly undominated offers and reservation prices can be characterized as follows.

Lemma 2. For an agent with money holdings $\eta \in \mathbb{R}_{+}$, the set of weakly undominated offers $\mathcal{A}(\eta)$ and the set of weakly undominated reservation prices $\mathcal{B}(\eta)$ are

$$
\begin{aligned}
& \mathcal{A}(\eta)=\left\{\begin{array}{c|c}
o \in \mathbb{R}_{+}^{*} & \begin{array}{r}
o \in \mathbb{R}_{+} \text {and }[\mathcal{V}(\eta+o)-c>\mathcal{V}(\eta) \text { or }[\mathcal{V}(\eta+o)-c \geq \mathcal{V}(\eta) \\
\text { and } \left.\left.\forall o^{\prime}>o \mathcal{V}(\eta+o)=\mathcal{V}\left(\eta+o^{\prime}\right)\right]\right] \text { and } \\
\left.\forall o^{\prime}<o\left[\mathcal{V}\left(\eta+o^{\prime}\right)-c \leq \mathcal{V}(\eta) \text { or } \mathcal{V}\left(\eta+o^{\prime}\right)<\mathcal{V}(\eta+o)\right]\right] \\
\text { or } \quad\left[o=\infty \text { and } \forall o^{\prime} \in \mathbb{R}_{+} \mathcal{V}\left(\eta+o^{\prime}\right)-c \leq \mathcal{V}(\eta)\right]
\end{array}
\end{array}\right\} \\
& \mathcal{B}(\eta)=\left\{r \in[0, \eta] \mid \begin{array}{c}
\left.\forall r^{\prime}>r u+\mathcal{V}\left(\eta-r^{\prime}\right) \leq \mathcal{V}(\eta)\right] \text { and }[u+\mathcal{V}(\eta-r) \geq \mathcal{V}(\eta) \\
\text { or } \left.\forall r^{\prime}<r\left[\exists o \in\left(r^{\prime}, r\right] u+\mathcal{V}(\eta-o)>\mathcal{V}(\eta)\right]\right]
\end{array}\right\} .
\end{aligned}
$$

The proof of Lemma 2 is given in Appendix A. Although the conditions that define $\mathcal{A}(\eta)$ and $\mathcal{B}(\eta)$ seem complicated, they essentially correspond to the intuitive no-regret criteria: weakly undominated offers are those that would not make a seller worse off in case a trade were to occur at that offer price, and weakly undominated reservation prices are those that would allow a buyer to accept an offer as long as it does not exceed his full valuation of a unit of the consumption good. The rest of the conditions are merely technical conditions that will be satisfied trivially in applications of the lemma. Given Lemma 2, the weakly undominated offer strategy $\omega$ and reservation-price strategy $\rho$ are defined as, for any $\eta \in \mathbb{R}_{+}, \omega(\eta) \in \mathcal{A}(\eta)$ and $\rho(\eta) \in \mathcal{B}(\eta)$.

To define the value function, consider an agent of type $i$ with money holdings $\eta$. The value of his money holdings $\mathcal{V}(\eta)$ depends on his potential trade meetings. With equal probability $1 / k$, the agent may meet a producer of his consumption good first or he may meet a consumer of his production good first. Given the discount rate $\gamma$, the random-matching frequency $\mu$, the reservation-price distribution $R$, and offer distribution $\Omega$, the value function can be written as follows:

$$
\begin{aligned}
\mathcal{V}(\eta)=\frac{\mu}{k \gamma+2 \mu} & \left(\max _{r \in \mathcal{B}(\eta)} \int_{0}^{r}(u+\mathcal{V}(\eta-x)-\mathcal{V}(\eta)) d \Omega(x)+\mathcal{V}(\eta)\right. \\
& \left.+\max _{o \in \mathcal{A}(\eta)}((1-R(o))(\mathcal{V}(\eta+o)-c-\mathcal{V}(\eta)))+\mathcal{V}(\eta)\right) .
\end{aligned}
$$


Equation (12) is the Bellman equation for $\mathcal{V}$. Note that the parameters $\mu, \gamma$, and $k$ function as a single parameter in the form of $k \gamma / \mu$. For simplicity, define $\phi \equiv k \gamma / \mu$. Note that $\phi>0$, and $1 / \phi$ can be regarded as the effective trading speed.

The state of the environment is summarized by the distribution of money holdings. Implicitly, the money holdings of each agent is a continuous-time, pure-jump Markov process on the state space $\mathbb{R}_{+}$. The environment is stationary if under the transition probabilities induced by the optimal trading strategy $(\omega, \rho)$, the distribution $H$ is a stationary initial distribution of this process.

Definition 1. A stationary Bayesian Nash equilibrium with weakly undominated strategies consists of a time-invariant profile $\langle H, R, \Omega, \omega, \rho, \mathcal{V}\rangle$ that satisfies

(a) $H$ is stationary under trading strategy $\omega$ and $\rho$, and the reservation-price distribution $R$ and the offer distribution $\Omega$ are derived from $H, \omega$, and $\rho$ according to (1) and (2).

(b) Given the distributions for money holdings $H$, reservation price $R$, and offer $\Omega$, the trading strategy is weakly undominated; $(\rho(\eta), \omega(\eta)) \in \mathcal{A}(\eta) \times \mathcal{B}(\eta)$ for all $\eta \in \mathbb{R}_{+}$, and value function $\mathcal{V}$ solves Bellman equation (12). That is,

$$
\begin{aligned}
\mathcal{V}(\eta)=\frac{1}{\phi+2}( & \int_{0}^{\rho(\eta)}(u+\mathcal{V}(\eta-x)-\mathcal{V}(\eta)) d \Omega(x)+\mathcal{V}(\eta) \\
& +(1-R(\omega(\eta)))(\mathcal{V}(\eta+\omega(\eta))-c-\mathcal{V}(\eta))+\mathcal{V}(\eta))
\end{aligned}
$$

Hereafter, I will refer to the equilibrium in Definition 1 as the stationary equilibrium. Bellman equation (12) implies the following property of the value function.

LEMMA 3. The value function $\mathcal{V}$ is bounded above by $\frac{u}{\phi}$ and below by 0.

The value function is nonnegative because agents' participation in trade is voluntary, and autarky yields a lifetime utility of zero. The upper bound is a consequence of the fact that the agents' consumption opportunities are bounded by the frequency of meetings. The maximum lifetime expected utility that an agent can obtain is by consuming at every meeting with a producer of his consumption good but never producing himself, which yields utility $\frac{u}{\phi}$.

\section{SINGLE-PRICE EQUILIBRIA}

Consider a potential stationary equilibrium at which all trades occur at a single price $p$. To characterize such an equilibrium, I first conjecture the strategy profile that gives rise to the equilibrium. Assuming that such a strategy is adopted, I will derive the stationary money-holdings 
distribution and solve for the corresponding value function. To study the existence of such an equilibrium, I will then provide a set of sufficient conditions under which the presumed trading strategy is optimal. Applying these conditions, I will show some examples of the equilibrium.

\subsection{Conjectured Equilibrium Strategy}

Consider the formulation of the stationary equilibrium above in the special case that all trades occur at price $p \in \mathbb{R}_{+}$. By definition, for any trade meeting in equilibrium, either the buyer is not willing to pay the seller's offer price or the buyer and the seller trade at exactly price $p$. Formally, let $\mathcal{H}$ denote the product measure on $\mathbb{R}_{+} \times \mathbb{R}_{+}$, with $H$ in each coordinate.

Definition 2. A stationary single-price equilibrium at which all trades occur at a finite price $p$ is a stationary equilibrium that satisfies an additional condition. For meetings between a buyer and a seller with money holdings $\left(\eta_{b}, \eta_{s}\right) \in \mathbb{R}_{+} \times \mathbb{R}_{+}$, the union of the following two events occurs with probability 1 (measurable with respect to $\mathcal{H}$ ): either $\rho\left(\eta_{b}\right)<\omega\left(\eta_{s}\right)$ so that trade does not occur or $\omega\left(\eta_{s}\right)=p \leq \rho\left(\eta_{b}\right)$ so that trade occurs at price $p$.

Literally, autarky is a single-price equilibrium - the price is infinity. To focus on the interesting monetary equilibria where there are active trades, I exclude autarky from the definition of singleprice equilibrium by limiting the effective prices at which trades are conducted to be finite.

If all trades occur at price $p$, the effective money holdings of an agent are those that are integer multiples of $p$. I consider money-holdings distributions only of this kind. That is, the support of the distribution $H$ is a subset of the discrete set $p^{\mathbb{N}} \equiv\{0, p, 2 p, \ldots\}$. For simplicity, define

$$
h(n) \equiv H(\{n p\}) .
$$

That is, $h(n)$ is the measure of the set of agents who hold precisely $n p$ units of money. Now, instead of working with the measure $H$ on $\mathbb{R}_{+}$, I work with the equivalent distribution $h$ on $\mathbb{N}$. I say that an agent is in state $n$ if his money holdings are $n p$.

For the equilibrium in question, money is valued and $p$ units of money are traded for one unit of a consumption good. An agent can obtain $p$ units of money only by selling his good, which costs c. An agent with a lot of money in hand, who is less worried about financing future consumption, may demand a higher price to compensate for his disutility of production than an agent with less money, and he may not make the sale at that higher price. This intuition suggests a potential endogenous upper bound on an agent's money holdings. The following proposition confirms such an intuition.

Proposition 1. There exists no single-price equilibrium at which agents' money holdings are unbounded. 
Proof. Suppose, to the contrary, that there exists a single-price equilibrium where all transactions occur and only occur at price $p$, and agents' potential money holdings are unbounded. At such an equilibrium, agents must be willing to sell at price $p$ regardless of their money holdings in order to accumulate an unlimited amount of money. This implies that for any $\eta \in \mathbb{R}_{+}, p \in \mathcal{A}(\eta)$. By Lemma 2, a necessary condition for $p \in \mathcal{A}(\eta)$ is that $\mathcal{V}(\eta+p)-\mathcal{V}(\eta) \geq c$. Such a condition can be satisfied only if $\mathcal{V}$ is unbounded given that $c>0$, which contradicts Lemma 3.

Note that Proposition 1 does not hold in GZ. When production is costless, the money-holdings distribution that is consistent with a single-price equilibrium must have infinite support, since agents always have an incentive to produce and acquire more money. Proposition 1 states that an infinite-support single-price equilibrium does not exist in a costly-production environment.

Let $N p$ ( $N$ units of $p$ ) denote the maximum quantity of money that an agent holds in equilibrium. The support of money-holdings distribution $H$ is $\{0, p, 2 p, \ldots, N p\}$. A conjectured optimal strategy profile for a single-price equilibrium is as follows. ${ }^{3}$

(i) All buyers with money holdings of at least $p$ accept offer $p$ :

$$
\forall n=1,2, \ldots \quad \rho(n p) \geq p .
$$

(ii) All sellers with money holdings of less than $N p$ offer price $p$ :

$$
\forall n=0,1,2, \ldots, N-1 \quad \omega(n p)=p .
$$

(iii) Agents with money holdings of greater than or equal to $N p$ offer above $p$ :

$$
\forall n \geq N \quad \omega(n p)>p .
$$

(iv) Offers made by agents with money holdings of $N p$ or more are not accepted by any buyer:

$$
\forall n \geq N \quad \omega(n p)>\max _{l \leq N} \rho(l p) .
$$

(v) Sellers in state $N$ offer to sell at $J p: \omega(N p)=J p$.

(vi) There exists a least-money balance $k p(K \geq N)$, buyers with money holdings above which are willing to accept offer $J p: \rho(n p) \geq J p$ if and only if $n>K$.

Note that there is a substantial amount of monotonicity embedded in the above trading strategy profile (i, iii, iv, and vi). Hereafter, I refer to a single-price equilibrium at which all trades occur at price $p$, the support of money-holdings distribution is $\{0, p, 2 p, \ldots, N p\}$, and all agents adopt the trading strategy profile (i) - (vi) as an $N-J-p$ equilibrium.

\subsection{Stationary Money-Holdings Distribution}

Assume that agents adopt the strategy profile described above. Let the proportion of agents who have positive money holdings be $m, m \equiv \sum_{n=1}^{N} h(n)$. Note that $h(0)=1-m$.

\footnotetext{
${ }^{3}$ There are certainly other single-price equilibria that satisfy Definition 2, in particular, equilibria with different strategies in states above $N$. For example, (iii) can be relaxed to allow agents with money holdings strictly higher than $N$ to offer $p$ again. In that case, the value functions and, hence, the equilibrium conditions, are more difficult to characterize.
} 
Since all transactions are made at price $p$, an agent moves into state $n$ only by making either a sale from state $n-1$ or a purchase from state $n+1$. He also moves out of state $n$ by making either a purchase or a sale. According to the presumed trading strategy, a type $i$ agent with a money balance less than $N p$ makes a sale whenever he meets an agent of type $i+1$ who has money. The Poisson frequency of such trading is $\mu m / k$. A type $i$ agent with money makes a purchase whenever he meets an agent of type $i-1$ who is willing to sell at price $p$ (all agents except those in state $N$ ), and the Poisson frequency of such trading is $\mu(1-h(N)) / k$. Stationarity requires that the time rate of inflow to any state $n$ equals the time rate of outflow from state $n$. Or, equivalently, the time rate of inflow into all the states above an arbitrary $n$ (state $n, n+1$ up to $N$ ) is equal to the time rate of outflow from these states. That is, for any $n=1,2, \ldots, N$,

$$
\frac{\mu m}{k} h(n-1)=\frac{\mu(1-h(N))}{k} h(n) \text {. }
$$

The size of the population in state $n$ can then be expressed as a function of the measures of agents having money $m$ and those holding the upper bound $h(N)$ : for any $n=0,1, \ldots, N$,

$$
h(n)=\left(\frac{m}{1-h(N)}\right)^{n}(1-m) .
$$

Note that equation (16) is consistent with the costless-production case of GZ, where $N=\infty$, $h(N)=0$, and the money-holdings distribution is geometric. The fact that (16) holds for $n=N$ implies that

$$
h(N)(1-h(N))^{N}=(1-m) m^{N} .
$$

The nominal money stock $M$ is the sum of money held by all agents. That is,

$$
M=\sum_{n=1}^{N} n p h(n)=p(1-m) \sum_{n=1}^{N} n\left(\frac{m}{1-h(N)}\right)^{n} .
$$

From equation (18), $h(N)$ can be solved as a function of the real-money balance $\frac{M}{p}$ :

$$
h(N)=\frac{m-(1-m) \frac{M}{p}}{m(N+1)-\frac{M}{p}} .
$$

For a given $N$, the real-money balance $\frac{M}{p}$ and the proportion of agents holding money $m$ have to jointly satisfy equations (17) and (19) as well as $0 \leq m \leq 1$ and $0 \leq h(N) \leq 1$. It can be shown that the solution exists, which, together with (16), defines the distribution completely. More specifically, the following lemma holds.

LEMma 4. A stationary money-holdings distribution characterized by $N, \frac{M}{p}, m$, and $h$ always exists, and it has the following features:

(a) The upper bound $N>\frac{M}{p}$. 
(b) Given $N, m$ is uniquely determined by $\frac{M}{p}$ and vice versa.

(c) The distribution $h$ satisfies the following:

- If $\frac{M}{p}<\frac{N}{2},\{h(n)\}_{n=0}^{N}$ is decreasing in $n$.

- If $\frac{M}{p}=\frac{N}{2}, h(n)=\frac{1}{N+1}$.

- If $\frac{M}{p}>\frac{N}{2},\{h(n)\}_{n=0}^{N}$ is increasing in $n$.

Lemma 4 asserts that for a given $N$, there is a one-to-one mapping between $m$ and $\frac{M}{p}$; therefore, either one uniquely determines the stationary distribution. Note that if the money-holdings distribution $h$ is decreasing in $n$, it resembles a censored-geometric distribution: the proportion of agents holding a higher quantity of money declines geometrically before it hits the upper bound. The proof of Lemma 4 is given in Appendix B.

\subsection{Value Function}

Next, I solve the equilibrium value function assuming that the presumed trading strategy is adopted. By the strategy profile (i) - (vi), agents in states in the support, except those in state $N$, offer to sell at exactly price $p$ :

$$
\Omega(p)=1-h(N) ; \quad \forall x<p \quad \Omega(x)=0 .
$$

Agents in state $N$ offer to sell at $J p$ :

$$
\forall p<x<J p \quad \Omega(x)=\Omega(p) ; \quad \forall x \geq J p \quad \Omega(x)=1 .
$$

All buyers with money holdings in the support accept offer $p$, not the higher offer $J p$ made by agents in state $N$ :

$$
R(p)=1-m ; \quad R(J p)=1 .
$$

For convenience, define $V(n)=\mathcal{V}(n p)$. Given the offer and reservation-price distributions satisfy (20) — (22), the presumed trading strategy profile (i) - (iv) implies that the value function (13) can be written as the following set of equations:

$$
V(0)=\frac{m}{\phi+m}(V(1)-c)
$$

for $n=1,2, \ldots, N-1$,

$$
V(n)=\frac{1}{\phi+m+1-h(N)}((1-h(N))(u+V(n-1))+m(V(n+1)-c))
$$

and

$$
V(N)=\frac{1-h(N)}{\phi+1-h(N)}(u+V(N-1)) .
$$


The above $N+1$ equations determine the value function for the states in the support, $n \leq N$. The system of equations (24) can be rewritten in the matrix form,

$$
\left(\begin{array}{c}
V(n+1) \\
V(n) \\
\chi
\end{array}\right)=\mathcal{T}\left(\begin{array}{c}
V(n) \\
V(n-1) \\
\chi
\end{array}\right)
$$

where $\chi=(1-h(N)) u-m c$, and

$$
\mathcal{T}=\left(\begin{array}{ccc}
\left(\frac{\phi}{m}+\frac{1-h(N)}{m}+1\right) & -\frac{1-h(N)}{m} & -\frac{1}{m} \\
1 & 0 & 0 \\
0 & 0 & 1
\end{array}\right)
$$

Equation (26) is a second-order linear difference equation. Its family of solutions is given in terms of eigenvectors of the matrix $\mathcal{T}$, which have three real eigenvalues: $\lambda_{0}=1, \lambda_{1} \in(0,1)$, and $\lambda_{2}>1$. The precise solution is determined by the two endpoint equations (23) and (25). Appendix $\mathrm{C}$ solves the difference equation in detail. To summarize the result, the value function $V$ takes the following form: for $n=0,1, \ldots, N$,

$$
V(n)=\theta_{0} / \phi+\theta_{1} \lambda_{1}^{n}+\theta_{2} \lambda_{2}^{n}
$$

where $\theta_{0}, \theta_{1}$, and $\theta_{2}$ are functions of $N, m, h(N)$, and the parameters of the model $u, c$, and $\phi$. The explicit functional forms for $\lambda_{1}, \lambda_{2}, \theta_{0}, \theta_{1}$, and $\theta_{2}$ are given in Appendix C.

The value function for states off the support, $n>N$, can be defined similarly, given (i) - (vi). For $n=N+1, \ldots, K$,

$$
V(n)=\frac{1-h(N)}{\phi+1-h(N)}(u+V(n-1))
$$

and for $n \geq K+1$,

$$
V(n)=\frac{1}{\phi+1}((1-h(N))(u+V(n-1))+h(N)(u+V(n-J))) .
$$

Given $\{V(n)\}_{n=0}^{N}$, the value function for $n>N$ can be solved recursively by equations (29) and (30). The following lemma can be shown from the explicit solution to the value function (28), (29), and (30).

Lemma 5. At an N-J-p equilibrium, the value function $V$ is strictly increasing in $n$.

Although the class of equilibria characterized has the feature that each trader's money holdings are always an integer multiple of $p$, the value function is defined for noninteger multiples as well. Given the presumed optimal trading strategy, the value function is evidently a step function. Specifically, if $[x]$ denotes the integer part of $x$ (that is, $x=[x]+\epsilon$ for some $\epsilon \in[0,1)$ ), then

$$
\mathcal{V}(\eta)=V([\eta / p]) .
$$


This completes the derivation of the value function.

\subsection{Equilibrium Strategy}

The objective of this section is to find the set of conditions under which the conjectured equilibrium trading strategy profile (i) - (vi) is weakly undominated as well as optimal.

I have shown that at a conjectured $N-J-p$ equilibrium, the value function $\mathcal{V}$ is a step function, continuous from the right, and strictly increasing at integer multiples of $p$. These properties can be used to simplify the set of weakly undominated trading strategies. It is easy to show that $\mathcal{V}$, being continuous from the right, reduces the set of weakly undominated reservation prices to

$$
\mathcal{B}(\eta)=\left\{r \in[0, \eta] \mid u+\mathcal{V}(\eta-r) \geq \mathcal{V}(\eta) \text { and } \forall r^{\prime}>r u+\mathcal{V}\left(\eta-r^{\prime}\right) \leq \mathcal{V}(\eta)\right\}
$$

Generically, this set contains a single point. In the exceptional case where $\mathcal{V}(\eta)-\mathcal{V}(\eta-r)$ is exactly $u, \mathcal{B}(\eta)$ is an interval, and buyers are indifferent with the reservation prices in this interval. In this case, I assume that buyers choose the maximum among the set of indifferent reservation prices. That is, the optimal weakly undominated reservation-price strategy is

$$
\rho(\eta)=\max \mathcal{B}(\eta)=\max \{r \in[0, \eta] \mid u+\mathcal{V}(\eta-r) \geq \mathcal{V}(\eta)\}
$$

This reservation-price strategy covers the generic case where $\mathcal{B}(\eta)$ is a single point as well as the special case when there is a tie. For the rest of the paper, I will focus on the $N-J-p$ equilibrium at which (33) is satisfied. Given that $\mathcal{V}$ is a step function and continuous from the right, by equation (33), for any money holdings $\eta \in \mathbb{R}_{+}$, the optimal reservation price $\rho(\eta)$ is an integer multiple of $p$.

Similarly, given the properties of the value function, it is easy to show that for any money holdings $\eta \in \mathbb{R}_{+}$, the set of weakly undominated offers can be reduced to

$$
\mathcal{A}(\eta)=\left\{\begin{array}{l|l}
o \in \mathbb{R}_{+}^{*} & \begin{array}{l}
{\left[\begin{array}{l}
o \in \mathbb{R}_{+} \text {and } \mathcal{V}(\eta+o)-c>\mathcal{V}(\eta) \text { and } o / p \text { is an integer }
\end{array}\right]} \\
\text { or }\left[o=\infty \text { and } \forall o^{\prime} \in \mathbb{R}_{+} \mathcal{V}\left(\eta+o^{\prime}\right)-c \leq \mathcal{V}(\eta)\right]
\end{array}
\end{array}\right\}
$$

and the optimal offer is given by

$$
\omega(\eta) \in \arg \max _{o \in \mathcal{A}(\eta)}\{(1-R(o))(\mathcal{V}(\eta+o)-c-\mathcal{V}(\eta))\} .
$$

To summarize,

Lemma 6. At an N-J-p equilibrium, the optimal reservation-price and offer strategy is given by (33) and (35). Furthermore, for any money holdings $\eta$, both $\rho(\eta)$ and $\omega(\eta)$ are integer multiples of $p$ with the exception that $\omega(\eta)=\infty$. 
In what follows, I will focus on the set of trading strategies that are integer multiples of $p$. I will look for the set of sufficient conditions under which the strategy profile (i) - (vi) for an $N-J-p$ equilibrium is indeed optimal. All conditions are stated in terms of the value function which can be expressed in the parameters of the model directly by applying the solutions to the value function (28), (29), and (30).

By the presumed optimal strategy (ii) and (iii), an agent's offer strategy changes distinctly at the money-holdings upper bound $N p$ : a seller offers to sell at price $p$ in state $N-1$ and is not willing to offer $p$ in state $N$. By the optimal offer strategy (35), such a change requires that

$$
\begin{aligned}
& V(N)-c>V(N-1) \\
& V(N+1)-c \leq V(N)
\end{aligned}
$$

given the reservation-price distribution (22). This pair of inequalities defines precisely the upper bound $N$ on money holdings as the point at which the incremental value of $p$ units of money drops below the cost of production $c$, and therefore, they are necessary for the existence of the singleprice equilibrium with upper bound $N$. Assuming that these two conditions hold, I now examine the rest of the strategy profile. First, these two conditions imply the following characteristics of the value function. Recall that $K$ is the state above which buyers accept offer $J p$ made by sellers in state $N, K \geq N$.

LEMma 7. At an N-J-p equilibrium, the value function $V$ is strictly concave in $n$ up to $K$. That is, for all $1 \leq n \leq K, V(n)-V(n-1)$ is decreasing in $n$.

Lemma 7 is proved in Appendix D. The value function may not be concave above $K$. Given Lemma 7, it is easy to show the existence of $K$ as a function of $J$. Note that if $J=\infty$, then $K=\infty$ since no agent with finite money holdings can afford an offer of infinity. Furthermore,

LEMMA 8. At an N-J-p equilibrium where $J$ is finite, there exists a finite $K$ such that buyers in states up to $K$ reject offer Jp and buyers in state $K+1$ accept offer Jp.

Proof. For a given finite $J>1$, if $V(J)-V(0) \leq u$, then $K=J-1$ by (33). So, $K$ is finite, and for $n \leq K<J$, agents in state $n$ cannot afford to pay Jp. Consider the case that $V(J)-V(0)>u$. Suppose $K$ is infinite; that is, no money holdings are high enough for agents to accept $J p$. By Lemma 7 , the value function is concave in $n$ throughout. The concavity and increasingness of $V$ in $n$ imply that for any finite $J>1$, there exists an $\hat{n} \geq J$ such that $V(\hat{n})-V(\hat{n}-J)>u$. By (29), it is easy to show that $V(n)-V(n-J) \rightarrow 0$ as $n \rightarrow \infty$. Therefore, there exists a threshold $n^{*}>\hat{n}$ such that $V\left(n^{*}\right)-V\left(n^{*}-J\right)>u$, but $V\left(n^{*}+1\right)-V\left(n^{*}+1-J\right) \leq u$. By (33), then, $\rho\left(\left(n^{*}+1\right) p\right) \geq J p$, which contradicts the supposition. By definition, $K=n^{*}$. That is,

$$
K=\max \{n \mid V(n)-V(n-J)>u\}
$$


which is finite and unique for a given $J$. By (33) and (38), for all $n \leq K, \rho(n p)<J p$, and $\rho((K+1) p) \geq J p$.

Lemma 9. Given that conditions (36) and (37) hold, it is optimal for agents with money holdings of at least $p$ to accept offer $p$.

Proof. By Lemma 7, conditions (36) and (37) guarantee that the value function is concave up to $K$. Consider a buyer in state $n$. If $n=1,(23)$ and (24) for $n=1$ imply that (1$h(N))(u+V(0)-V(1))=\phi(V(1)-V(0))+m((V(1)-V(0))-(V(2)-V(1)))$, which is positive, since $V$ is increasing and concave in $n .{ }^{4}$ So, $u+V(0)>V(1)$. If $2 \leq n \leq K$, by concavity, $V(n)-V(n-1)<V(1)-V(0)<u$. Therefore, $u+V(n-1)>V(n)$. If $n \geq K+1$, from (30), $u+V(n-1)-V(n)=\phi V(n)+h(N)(V(n)-V(n-J))$, which is positive, since $V$ is increasing. So, $u+V(n-1)>V(n)$. In all three cases, by $(33), \rho(n p) \geq p$ for all $n \geq 1$. That is, it is optimal for buyers with money holdings of at least $p$ to set their reservation prices no lower than $p$.

Lemma 9 confirms that the proposed reservation-price strategy (i) is optimal. The optimality of the reservation-price strategy with respect to the other equilibrium offer $J p$ requires another condition, which is also a necessary condition for an $N-J-p$ equilibrium. Define $J^{*}$ as the minimum value of $J$ such that $J p \in \mathcal{A}(N p)$,

$$
J^{*}=\min \{J \mid V(N+J)-c>V(N)\} .
$$

By Lemma 5 and $(37), J^{*} \geq 2$. Note that $J^{*}$ can be infinity.

Lemma 10. At an N-J-p equilibrium, the following condition must hold:

$$
\text { either } \quad N<J^{*} \quad \text { or } \quad \text { if } N \geq J^{*} \text {, then } V(N)-V\left(N-J^{*}\right)>u \text {. }
$$

Proof. Suppose not. That is, $N \geq J^{*}$ and $V(N)-V\left(N-J^{*}\right) \leq u$. Then, by (33), $\rho(N p) \geq J^{*} p$, which implies that $1-R\left(J^{*} p\right)=h(N)>0$. If $J=J^{*}$, then trades are occurring at both price $p$ and $J^{*} p$, which contradicts the definition of single-price equilibrium. Consider the case where $J>J^{*}$. Denote the net expected value of an agent in state $n$ offering $j p$ by $W(n, j)$ :

$$
W(n, j)=(1-R(j p))(V(n+j)-c-V(n)) .
$$

Then, $W\left(N, J^{*}\right)=h(N)\left(V\left(N+J^{*}\right)-c-V(N)\right)>0=W(N, J)$, since $R(J p)=1$. That is, an agent in state $N$ can do strictly better by deviating from offering $J p$ and offering $J^{*} p$ instead, which contradicts the fact that $\omega(N p)=J p$ is an equilibrium strategy.

\footnotetext{
${ }^{4}$ In the special case where $N=K=1,(1-h(N))(u+V(0)-V(1))=\phi V(1)>0$. No concavity condition is needed here.
} 
Condition (40) eliminates all effective higher values of reservation price and thus ensures that $R(x)=1$ for any potential offer $x>p$ by sellers in state $N$. A weaker condition $V(N)-V(N-J)>u$ (no agent in state $N$ is willing to pay $J p$ ) is not enough to guarantee (iv), since if there are agents willing to pay some $j p$ which is above $J^{*} p$ but below $J p$, it is not the best response for agents holding $N p$ units of money to offer $j p$. The next lemma, which is a direct result of the concavity of the value function and condition (40), guarantees the validity of the reservation-price strategy (iv).

LEMma 11. If conditions (36), (37), and (40) hold, it is optimal for buyers with money holdings in the support not to accept offer $J^{*} p$ or higher.

The proof of this lemma is omitted since the idea is very simple: if agents with money holdings $N p$ do not or can not accept offer $J^{*} p$ or higher, agents with money holdings less than $N p$ do not or can not accept those offers either. The last part of the reservation-price strategy to be checked is (vi), half of which-buyers with money holdings no more than $k p$ do not accept offer $J p$ - has been proved in Lemma 8. The following lemma verifies the optimality of the other half of strategy (vi). The proof is given in Appendix E.

LEMMA 12. If conditions (36) and (37) hold, it is optimal for buyers with money holdings of greater than $\mathrm{kp}$ to accept an offer of Jp.

Next, I examine the validity of offer strategy profile (ii), (iii), and (v) as an equilibrium offer strategy. Note that by the definition of $N-J-p$ equilibrium, sellers in state $N$ offer to sell at $J p(\mathrm{v})$ if and only if

$$
[J \in \mathbb{N} \text { and } V(N+J)-c>V(N)] \text { or }[J=\infty \text { and } \forall j \in \mathbb{N} V(N+j)-c \leq V(N)] .
$$

LEMMA 13. If conditions (36), (37), and (40) hold, then it is optimal for sellers with money holdings of less than $\mathrm{Np}$ to offer $p$.

Proof. By $(40)$, for all $j>1, R(j p)=1$. Consider a seller with money holdings of $n p$. Since $V(N)-V(N-1)>c$, by concavity, $V(n)-V(n-1)>c$ for all $n=1,2, \ldots, N$. Given the reservation-price distribution $R$, by (41), for any $n<N$ and $j>1, W(n, 1)=m(V(n+1)-c-$ $V(n))>W(n, j)=0>W(n, 0)$. Thus, for all $n<N, \omega(n p)=p$. That is, all agents holding less than $N p$ units of money should offer exactly $p$.

LEMMA 14. Given that conditions (36), (37), and (40) hold, if

$$
\forall n \in[N+\bar{l}-1, N+J) \quad V(n+1)-V(n) \leq c
$$

where $\bar{l}=\max \left\{K+1-N, J^{*}\right\}$, then it is optimal for sellers with money holdings of at least $N p$ to offer a price above $p$. 
Condition (43) is vacuous if $\bar{l}>J$. This extra condition is imposed because of the potential nonconcavity of the value function above $K$. It is a sufficient condition to ensure that agents in states between $N+\bar{l}-1$ and $N+J$ do not offer $p$. The proof is given in Appendix F.

The following proposition summarizes the above discussion and categorizes the various conditions into necessary and sufficient conditions for the existence of an $N-J-p$ equilibrium.

Proposition 2. For a given set of the parameters $M, u, c$, and $\phi$ :

(a) If an N-J-p equilibrium exists, then conditions (36), (37), (40), and (42) hold.

(b) If conditions (36), (37), (40), (42), and (43) hold, then an N-J-p equilibrium exists.

Note that if an $N-J-p$ equilibrium exists, generically, all conditions hold with strict inequality. Then, for the same money-holdings upper bound $N$, these conditions also hold for prices in a neighborhood of $p$. Varying the price continuously within the neighborhood yields a continuum of $N-J-p^{\prime}$ equilibria with $p^{\prime} \neq p$. That is, if one single-price equilibrium exists, a continuum of similar single-price equilibria with different prices exists in general. This partly answers one of the questions posed in the beginning of the paper: the existence of a continuum of single-price equilibria is robust to the introduction of costly production, conditional on the existence of one such equilibrium.

At an $N-J-p$ equilibrium with a finite $J$, the set of weakly undominated strategies $\mathcal{A}(N p)$ contains all integers above $J$, and sellers in state $N$ are indifferent among all the offers in $\mathcal{A}(N p)$. Thus, there is potentially an infinite number of outcome-equivalent single-price equilibria that differ only in the offer $J p$ that sellers with money holdings $s p$ make. Among these potential equilibria, one is of particular interest - the $N-J^{*}-p$ equilibrium. At this equilibrium, sellers in state $N$ offer the minimum price that they are willing to sell. Such an offer can be selected from the infinite set $\mathcal{A}(N p)$ among which agents in state $N$ are indifferent by adopting a stronger form of equilibrium refinement-Myerson's [7] properness in essence - on the offer strategy. ${ }^{5}$ The idea of the refinement is simple. Instead of assuming that buyers always accept offers below their reservation price and never accept offers above it, assume that they might make mistakes and that the chance of a small mistake is bigger than the chance of a big mistake. This can be done by having the tremble probability be a function of the net gains from trade. Given such a tremble strategy of the buyers, the sellers in state $N$ maximize the expected net payoff or, equivalently, maximize trading opportunity by making the lowest possible offer $J^{*} p$. Taking the tremble probability to zero, I get back the $N-J^{*}-p$ equilibrium. The details of the refinement process are omitted here.

\footnotetext{
${ }^{5}$ One can study a full-blown single-price proper equilibrium at which both reservation-price strategy and offer strategy are treated equally with the refinement process. In this sense, the refinement on offer strategy alone is not exactly Myerson's [7] properness.
} 
In what follows, I will look at some specific examples of the $N-J^{*}-p$ equilibria.

\subsection{Examples of $N-J^{*}-p$ Equilibria}

Conceptually, given a set of parameters of the model, one can apply Proposition 2 to check if an $N-J-p$ equilibrium exists. However, the existence conditions are usually difficult to study analytically, because these conditions expressed in terms of the solution to the value function are actually functions of the parameters of the model, which are polynomials of order at least $N^{2}$, and functions of the money-holdings distribution, which in general does not have an explicit solution. In the following, I study one simple equilibrium in which the existence conditions are easy to analyze - the $1-\infty^{*}-p$ equilibrium. ${ }^{6}$ This equilibrium provides many insights into the single-price equilibrium in general. In particular, such an equilibrium can be easily compared with those in the existing literature where a unit-inventory constraint is exogenously imposed. The elimination of an exogenous constraint on money holdings implies additional equilibrium existence conditions, but the welfare implication is the same to the extent that the equilibrium exists. For equilibria with higher value of $N$, I resort to numerical examples. I will show some examples of $N-2^{*}-p$ equilibria.

\section{The 1- $\infty^{*}-p$ Equilibrium}

Consider a potential $1-\infty^{*}-p$ equilibrium where all trades occur at price $p$, and all traders hold either no money (in which case they cannot buy) or $p$ units of money (in which case they choose not to sell). If such an equilibrium exists, the stationary money-holdings distribution takes the simple form:

$$
h(1)=m=\frac{M}{p} \quad \text { and } \quad h(0)=1-\frac{M}{p}
$$

and the value function can be solved from (28) and (29). Among the equilibrium conditions, condition (37) is implied by condition (42) given $J^{*}=\infty$, condition (40) holds trivially since $N=1$, and condition (43) is irrelevant since $K=\infty$. Then, by Proposition 2, a 1- $\infty^{*}-p$ equilibrium exists if and only if conditions (36) and (42) are satisfied. Condition (36) can be reduced to

$$
\frac{M}{p}<\Psi_{1} \equiv 1-\frac{\phi}{\left(\frac{u}{c}-1\right)}
$$

which requires $\phi+1<\frac{u}{c}$ for $\Psi_{1}>0$. Condition (42), given $J^{*}=\infty$ and $N=1$, is equivalent to

$$
\frac{u}{\phi}-V(1) \leq c
$$

\footnotetext{
${ }^{6}$ Note that for any given $N$ and $p$, if the set of weakly undominated offers $\mathcal{A}(N p)$ contains a finite $J$, then $\infty$ as an offer is weakly dominated. That is, there may exist a single-price equilibrium with $J=\infty$ only if $J^{*}=\infty$.
} 
( $\frac{u}{\phi}$ is the upper bound on the value function). Condition (46) is satisfied if and only if $\frac{u}{c}<\phi(\phi+1)$ and

$$
\frac{M}{p} \leq \Psi_{2} \equiv \frac{1}{2\left(\frac{u}{c}-1\right)}\left(-\frac{u}{c}(\phi-1)-1+\sqrt{\left(\frac{u}{c}(\phi-1)+1\right)^{2}-4\left(\frac{u}{c}-1\right)\left(\frac{u}{c}-\phi(\phi+1)\right)}\right)
$$

where $\Psi_{2}>0$ when $\frac{u}{c}<\phi(\phi+1)$. For both conditions (36) and (42), then, $\frac{u}{c} \in(\phi+1, \phi(\phi+1))$ which is nonempty if and only if $\phi>1$. It is easy to verify that when $\frac{u}{c}<2 \phi, \Psi_{1}<\Psi_{2}$, and when $\frac{u}{c} \geq 2 \phi, \Psi_{1} \geq \Psi_{2}$. Then, the price $p$ can be chosen such that when $\frac{u}{c} \in(\phi+1,2 \phi)$, $\frac{M}{p} \in\left(0, \Psi_{1}\right)$; when $\frac{u}{c} \in[2 \phi, \phi(\phi+1)), \frac{M}{p} \in\left(0, \Psi_{2}\right]$, then the corresponding equilibrium is a $1-\infty^{*}-$ $p$ equilibrium. In fact, since conditions (36) and (42) are the necessary and sufficient conditions for the equilibrium, the continuum of equilibria, indexed by the real-money balance $\frac{M}{p} \in\left(0, \Psi_{1}\right)$ or $\frac{M}{p} \in\left(0, \Psi_{2}\right]$ depending on the value of $\frac{u}{c}$, is the complete set of $1-\infty^{*}-p$ equilibria. Thus, I conclude the following result.

Corollary 2.1. For a given set of the parameters $M, u, c$, and $\phi, a 1-\infty^{*}-p$ equilibrium exists if and only if $\phi>1, \frac{u}{c} \in(\phi+1, \phi(\phi+1))$, the real-money balance $\frac{M}{p} \in\left(0, \Psi_{1}\right)$ when $\frac{u}{c} \in(\phi+1,2 \phi)$, and $\frac{M}{p} \in\left(0, \Psi_{2}\right]$ when $\frac{u}{c} \in(2 \phi, \phi(\phi+1))$.

Unlike GZ where a single-price equilibrium always exists for any parameters of the model, here it may exist only if $\phi<\frac{u}{c}$ (or the difference between the upper bound and the lower bound $\frac{u}{\phi}$ is greater than the production cost $c$ ). If $\phi$ is too high, or equivalently, the effective trading speed is too slow (small $\mu$, or large $k$, or large $\gamma$ ), it may not be worthwhile for a seller to produce one unit of his production good and incur the immediate cost in exchange for $p$ units of money to be used for consumption far into the future. For the particular $1-\infty^{*}-p$ equilibrium, the existence condition is tighter. Corollary 2.1 states that in addition to a tighter upper bound, $\phi$ cannot be too low $\left(\phi \in\left[\frac{1}{2}\left(\sqrt{1+4 \frac{u}{c}}-1\right), \frac{u}{c}-1\right)\right)$ for a given $\frac{u}{c}$. If $\phi$ is too low, or equivalently, the effective trading speed is too fast, the value of holding more money is high, since trading is relatively easy, such that agents with $p$ units of money will be willing to produce at finite prices. Similarly, for the equilibrium to exist, the relative gain of consumption over production $\frac{u}{c}$ cannot be too high or too low either for a given $\phi$. If $\frac{u}{c}$ is too low, it is not worth the effort to produce, and if $\frac{u}{c}$ is too high, the seller with $p$ units of money will be tempted to produce at other finie prices.

For a fixed price $p$, if one regards $p$ units of nominal divisible money (or one unit of real divisible money) as one unit of indivisible money, the $1-\infty^{*}-p$ equilibrium coincides with the monetary equilibria investigated in many previous studies with indivisible money and an exogenous upper bound on holdings of money (see, for example, Kiyotaki and Wright [4] and [5], KW hereafter). Viewing the equilibrium this way, I can compare the effect of an endogenous versus exogenous upper bound on money holdings in isolation of the divisibility of money. One main difference is in the equilibrium existence region. With the exogenous bound of one unit of indivisible money, 
KW requires only condition (36), or equivalently, $\frac{u}{c}>\phi+1$ and $\frac{M}{p}<\Psi_{1}$ for the existence of a monetary equilibrium. The rest of the conditions in Corollary 2.1, derived from (42), would be irrelevant, since no agent is able to carry more money. The endogenous upper bound $N=1$ results from the fact that it is suboptimal for an agent with $p$ units of money to sell a unit of his production good, since the value of any additional money $\frac{u}{\phi}-V(1)$ is no higher than the cost of producing the good $c$. Therefore, when the inventory constraint on holdings of money is eliminated, the equilibrium existence region may shrink.

Despite the difference in the equilibrium existence region, the welfare implication of the present model is in agreement with that of $\mathrm{KW}$. In the present model, the average welfare of a 1-J-p equilibrium can be computed as

$$
\text { welfare }=h(0) V(0)+h(1) V(1)=\frac{1}{\phi} \frac{M}{p}\left(1-\frac{M}{p}\right)(u-c) .
$$

Therefore, among the continuum of equilibria, the closer is the real-money balance $\frac{M}{p}$ (or the proportion of agents holding money $m$ ) to $1 / 2$, the higher is the average equilibrium welfare. This result, however, contradicts the welfare result in GZ's costless-production case, at which welfare is monotonically increasing in the real-money balance. For the particular $1-\infty^{*}-p$ equilibrium, it is easy to verify that given conditions on $\phi$ and $\frac{u}{c}$ are satisfied, both $\Psi_{1}$ and $\Psi_{2}$ are less than $1 / 2$. That is, among the continuum of $1-\infty^{*}-p$ equilibria that exists, it is true that welfare is monotonically increasing in the real-money balance. But in general, when the existence region for the real-money balance of an arbitrary 1- $J$ - $p$ equilibrium contains $1 / 2$, the welfare is not monotone. The welfare loss from a higher real-money balance comes from the slowing down of trade caused by the fact that agents with money holdings of $p$ do not produce. The cost of production, that is, the cost of obtaining money, induces agents to economize on the amount of money to hold, which simultaneously shrinks the productive population as well. In this respect, although the result technically coincides with $\mathrm{KW}$, the economic rational for too high a real balance to reduce welfare is more persuasive here since it is an outcome of an endogenous decision rather than the result of an exogenous constraint as in KW.

Numerical Examples of $N-2^{*}-p$ Equilibria

Single-price equilibria with higher value of money-holdings upper bound $N$ exist as well, although they are difficult to study analytically. In the following, I show a few numerical examples of $N-2^{*}-p$ equilibria. Since the $N-2^{*}-p$ equilibrium is just one of the $N-J-p$ equilibria, the discussion applies to the more general $N-J-p$ equilibria.

Figure 1 illustrates the existence region of $N-2^{*}-p$ equilibria for different $N$ in terms of the proportion of agents holding money $m$ for an environment with $u=1, c=0.5$, and $\phi=0.1$. The 
existence region expressed in $m$ can be easily translated into that in $\frac{M}{p}$ by Lemma 4 . Each thin line represents the value that $m$ can take, namely, the unit interval, from the left endpoint 0 to the right endpoint 1. The heavy bar on top of each thin line indicates the interval of $m$, where Proposition 2 guarantees existence of equilibria in each case. For example, the bottom line shows that the $1-2^{*}-p$ equilibrium exists for $m \in[0.61,0.9$ ). (The heavy bar runs from 0.61 to 0.9 .) Since the equilibrium conditions in Proposition 2 are only sufficient, the existence region may be larger than what is shown in the figure.

Figure 1. Equilibrium Existence Region for $m$

$$
(u=1, c=0.5, \phi=0.1)
$$

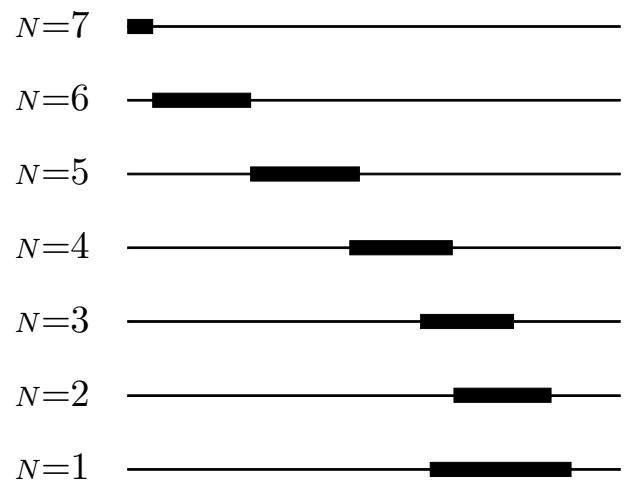

Figure 1 shows a two-dimensional multiplicity of the single-price equilibrium. One dimension is in $m$ (or real-money balance $\frac{M}{p}$ ): for each $N$ at which a single-price equilibrium exists, the existence region in $m$ is a continuum. Another dimension is in $N$ : equilibria with different moneyholdings upper bounds and, therefore, different money-holdings distributions coexist. For this particular example, coexisting equilibria range from those with more equally distributed money holdings, where $90 \%$ of agents hold $p$ units of money, to those with very concentrated money holdings, where $\epsilon \%$ ( $\epsilon$ arbitrarily small) of agents hold all the money in the economy and the "richest" agents hold $7 p$ units of money. The second dimension of multiplicity is new and not a feature of the single-price equilibrium in the costless-production environment of GZ.

Figure 2 shows the distributions of money holdings $h$ and associated welfare for four coexisting single-price equilibria with different money-holdings upper bounds $N=2,3,4,5$, but identical real-money balance $\frac{M}{p}=1.5$ in an environment where $u=1, c=0.5$, and $\phi=1 / 14$. As suggested by Lemma 4, the money-holdings distribution is increasing in $n$ for $N=2$, uniform for $N=3=2 \frac{M}{p}$, and decreasing in $n$ for $N=4,5$. In this particular example, welfare is increasing in the money-holdings upper bound $N$, but it is not true in general. There are cases where welfare is 
higher with a lower value of $N$, holding $\frac{M}{p}$ constant. The welfare of an equilibrium is determined by its money-holdings distribution, in particular, the measure of the unproductive population $h(N)$ and that of the pennyless population $h(0)$. Increasing the money-holdings upper bound $N$ has simultaneously the welfare-enhancing effect of reducing the former and the welfare-worsening effect of increasing the latter. It is possible for either effect to dominate.

Figure 2. Distribution $h$ of Coexisting Equilibria

$$
\left(u=1, c=0.5, \phi=1 / 14, \frac{M}{p}=1.5\right)
$$
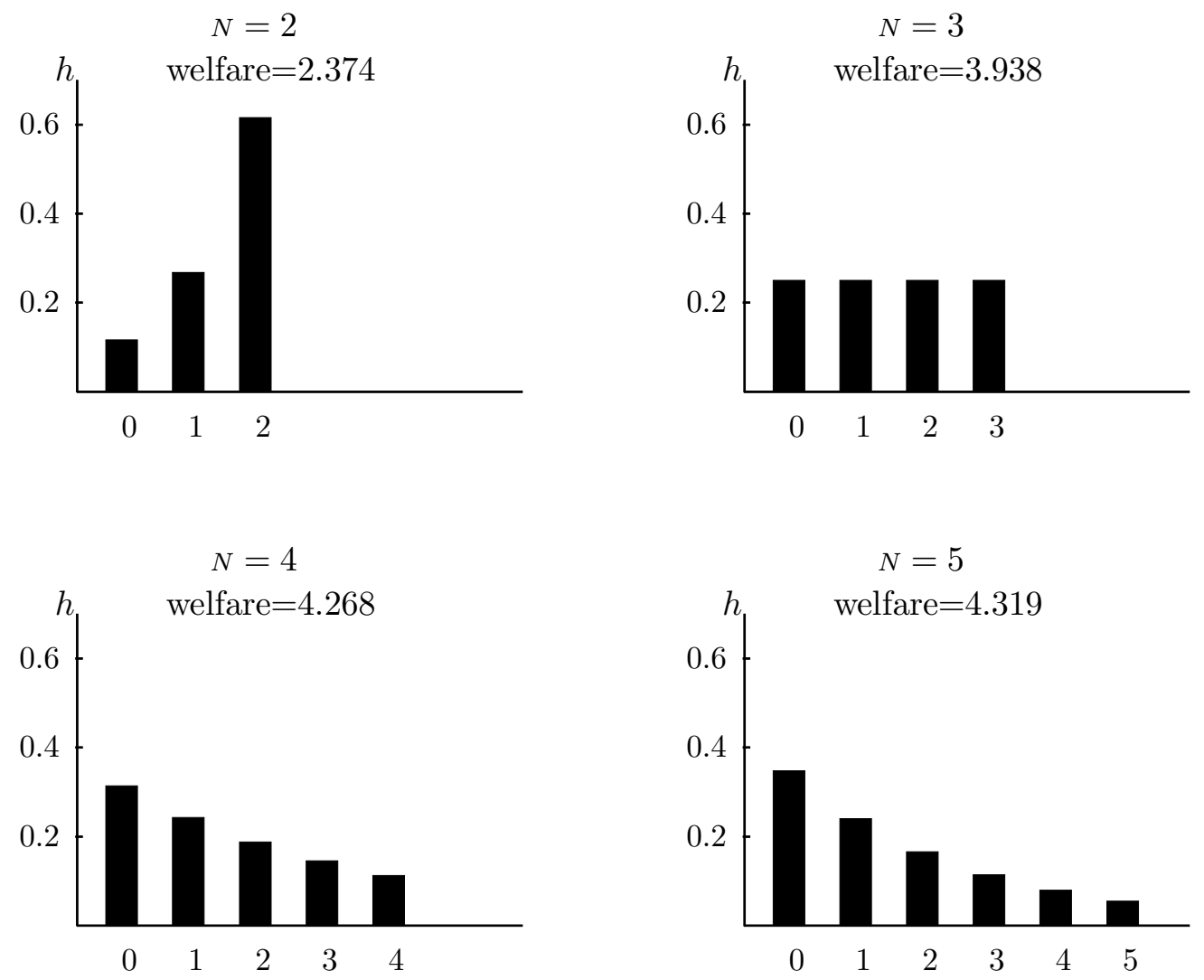

The coexistence of equilibrium allocations of consumption with different welfare levels in an environment with identical real-money balances demonstrates the importance of the distribution of money holdings in determining the equilibrium outcome. It is the entire distribution of money holdings, not the aggregate real-money balance, that provides the summary statistic of trading opportunities. The result suggests that for a given aggregate real-money balance, the equilibrium velocity of money is endogenously determined, and it may not be unique. It is through the velocity that the distribution of money as an important aspect of the environment plays out its role in shaping economic activities. 


\section{CONCLUDING REMARKS}

I have studied a random-matching model with divisible money and costly production. The model shares the main feature of the costless-production case studied in GZ regarding the existence of a continuum of single-price equilibria. I show that the phenomenon is robust to the introduction of costly production.

The mechanism through which the single-price equilibrium is reached in this costly-production environment is somewhat different from that of the costless-production model, although in both environments, the self-fulfilling belief of the prevailing price plays the crucial role. In GZ, sellers prefer posting the prevailing price because a higher offer reduces potential trading opportunities too much relative to the gain of obtaining more money. Here, in addition to the potential loss of buyers, the cost of production is also a deciding factor in sellers' offer decision. For those sellers whose money holdings reach a certain level, although they are reluctant to lose potential buyers, they may have no choice but to post a higher offer because the prevailing price is not enough to compensate for the cost of production.

A natural question that arises when comparing the single-price equilibria in the two models is whether a given single-price equilibrium in GZ is the limit of a sequence of $N-J-p$ equilibria as the cost goes to zero, or the converse, whether a sequence of $N-J-p$ equilibria converges to a GZ single-price equilibrium. I do not have answers to these questions, which of course would depend on the metric of convergence.

The results of this model have some bearing on the long-lasting debate regarding the neutrality of money. If one defines neutrality by the classical homogeneity property, which states that an increase in the quantity of money and an equiproportional change in price preserve stationary equilibrium (see Gale's [2] discussion of Patinkin), then such neutrality is supported by the present model. However, if one interprets neutrality by the invariance principle, which asserts that the set of stationary equilibria is at most finite and is invariant to changes in the money stock (Gale $[2]$ ), then the existence of a continuum of stationary equilibria is obviously in disagreement with this version of the neutrality, although the set of stationary equilibria is invariant to the nominal money stock. The present model lacks a sound equilibrium selection criterion in the context of a continuum of equilibria and a technical framework to study the dynamics of the system to address the neutrality-of-money question seriously. The effect of an increase in nominal money stock may depend on the way the increase is made, for example, its immediate impact on the distribution of money holdings, and the belief regarding the stationary equilibrium that the economy may evolve to, as well as the equilibrium path it may take. The present model cannot rule out the possibility that the economy might settle down anywhere within the continuum of equilibria, not necessarily at the one with only an equiproportional change in price and no change in distribution and real 
allocations.

Dynamic analysis may also provide an answer to the question of whether the continuum of stationary equilibria is all reachable from any initial distribution of money holdings. ${ }^{7}$ It is possible that the set of equilibrium paths from any given initial state is finite.

The current analysis has many other limitations. An important one is the focus on the singleprice stationary equilibrium. Such an equilibrium is chosen as a starting point for its relative simplicity and its resemblance to the standard Walrasian competitive equilibrium. However, even if the assumptions on the indivisibility of production and cost are maintained, I am confident that there exist stationary price-dispersion equilibria. One conjecture is that as trading "frictions" decline (trading becomes fast and time preference attenuates), the magnitude of price dispersion also would shrink, and in the limit, a price-dispersion equilibrium converges to a single-price equilibrium.

Another limitation of the present model is the restrictions on goods. The easiest way to relax such restrictions is to adopt the Shi [9] and Trejos and Wright [10] assumption, that is, to allow agents to produce divisible perishable goods according to a smooth production-cost function. ${ }^{8}$ Under such a setup, the existence of the single-price equilibrium and, furthermore, the existence of a continuum of whatever stationary equilibrium may exist are interesting questions to study. Future research will shed some light on these questions.

\footnotetext{
${ }^{7}$ This is an important point made by Wallace [11].

${ }^{8}$ Molico [6] studies such a model but only numerically under a different information assumption and a different trading protocol than mine.
} 


\section{APPENDIX}

\section{A. The Proof of Lemma 2}

Consider the offer first: $o \in \mathcal{A}(h) \Longleftrightarrow o$ is weakly undominated.

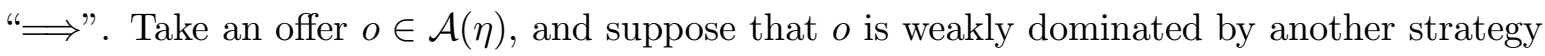
$o^{\prime}$. If $o \in \mathbb{R}_{+}$and $o^{\prime}<o$, then either (a) $\mathcal{V}\left(\eta+o^{\prime}\right)-c \leq \mathcal{V}(\eta)$ or $(\mathrm{b}) \mathcal{V}\left(\eta+o^{\prime}\right)<\mathcal{V}(\eta+o)$. Consider case (a) $\mathcal{V}\left(\eta+o^{\prime}\right)-c \leq \mathcal{V}(\eta)$ first. For all $r<o^{\prime}, \Pi_{s}\left(\eta, o^{\prime}, r\right)=\Pi_{s}(\eta, o, r)=0$. For every $r \in\left[o^{\prime}, o\right), \Pi_{s}\left(\eta, o^{\prime}, r\right)=\mathcal{V}\left(\eta+o^{\prime}\right)-c-\mathcal{V}(\eta) \leq 0=\Pi_{s}(\eta, o, r)$. For all $r \geq o$, $\Pi_{s}\left(\eta, o^{\prime}, r\right)=\mathcal{V}\left(\eta+o^{\prime}\right)-c-\mathcal{V}(\eta) \leq \mathcal{V}(\eta+o)-c-\mathcal{V}(\eta)=\Pi_{s}(\eta, o, r)$ since $\mathcal{V}$ is weakly increasing. That is, there does not exist an $r$ such that $\Pi_{s}\left(\eta, o^{\prime}, r\right)>\Pi_{s}(\eta, o, r)$. Therefore, offer $o$ is not weakly dominated by $o^{\prime}$. For case (b), since $\mathcal{V}\left(\eta+o^{\prime}\right)<\mathcal{V}(\eta+o)$, for all $r \geq o, \Pi_{s}\left(\eta, o^{\prime}, r\right)=$ $\mathcal{V}\left(\eta+o^{\prime}\right)-c-\mathcal{V}(\eta)<\mathcal{V}(\eta+o)-c-\mathcal{V}(\eta)=\Pi_{s}(\eta, o, r)$. Then, offer $o$ is not weakly dominated by $o^{\prime}$.

If $o \in \mathbb{R}_{+}$and $o^{\prime}>o$, then either (a) $\mathcal{V}(\eta+o)-c>\mathcal{V}(\eta)$ or (b) $\mathcal{V}(\eta+o)-c \geq \mathcal{V}(\eta)$ and $\mathcal{V}\left(\eta+o^{\prime}\right)=\mathcal{V}(\eta+o)$. For case (a), since $\mathcal{V}(\eta+o)-c>\mathcal{V}(\eta)$, for all $r \in\left[o, o^{\prime}\right), \Pi_{s}(\eta, o, r)=$ $\mathcal{V}(\eta+o)-c-\mathcal{V}(\eta)>0=\Pi_{s}\left(\eta, o^{\prime}, r\right)$. Therefore, offer $o$ is not weakly dominated by $o^{\prime}$. Consider case (b) $\mathcal{V}(\eta+o)-c \geq \mathcal{V}(\eta)$ and $\mathcal{V}\left(\eta+o^{\prime}\right)=\mathcal{V}(\eta+o)$. For all $r<o, \Pi_{s}\left(\eta, o^{\prime}, r\right)=\Pi_{s}(\eta, o, r)=0$; for all $r \in\left[o, o^{\prime}\right), \Pi_{s}(\eta, o, r)=\mathcal{V}(\eta+o)-c-\mathcal{V}(\eta) \geq 0=\Pi_{s}\left(\eta, o^{\prime}, r\right)$; and for all $r \geq o^{\prime}$, $\Pi_{s}(\eta, o, r)=\mathcal{V}(\eta+o)-c-\mathcal{V}(\eta)=\mathcal{V}\left(\eta+o^{\prime}\right)-c-\mathcal{V}(\eta)=\Pi_{s}\left(\eta, o^{\prime}, r\right)$. That is, there does not exist an $r$ such that $\Pi_{s}\left(\eta, o^{\prime}, r\right)>\Pi_{s}(\eta, o, r)$. Therefore, offer $o$ is not weakly dominated by $o^{\prime}$.

If $o=\infty$ and $\mathcal{V}\left(\eta+o^{\prime}\right)-c \leq \mathcal{V}(\eta)$, then for all $r<o^{\prime}, \Pi_{s}\left(\eta, o^{\prime}, r\right)=\Pi_{s}(\eta, o, r)=0$, and for every $r \in\left[o^{\prime}, \infty\right), \Pi_{s}\left(\eta, o^{\prime}, r\right)=\mathcal{V}\left(\eta+o^{\prime}\right)-c-\mathcal{V}(\eta) \leq 0=\Pi_{s}(\eta, o, r)$. That is, there does not exist an $r$ such that $\Pi_{s}\left(\eta, o^{\prime}, r\right)>\Pi_{s}(\eta, o, r)$. Therefore, offer $o$ is not weakly dominated by $o^{\prime}$.

"£" Take a weakly undominated offer $o$, and suppose that $o \notin \mathcal{A}(\eta)$. Then, any one of the following four cases may occur, and I will consider each separately. The first three cases deal with finite $o$, and the last case is for $o=\infty$.

(a) $o \in \mathbb{R}_{+}$and $\mathcal{V}(\eta+o)-c<\mathcal{V}(\eta)$. Take an $o^{\prime}>o$. Then, for all $r<o, \Pi_{s}\left(\eta, o^{\prime}, r\right)=$ $\Pi_{s}(\eta, o, r)=0$. For all $r \in\left[o, o^{\prime}\right), \Pi_{s}(\eta, o, r)=\mathcal{V}(\eta+o)-c-\mathcal{V}(\eta)<0=\Pi_{s}\left(\eta, o^{\prime}, r\right)$. And for all $r \geq o^{\prime}, \Pi_{s}(\eta, o, r)=\mathcal{V}(\eta+o)-c-\mathcal{V}(\eta) \leq \mathcal{V}\left(\eta+o^{\prime}\right)-c-\mathcal{V}(\eta)=\Pi_{s}\left(\eta, o^{\prime}, r\right)$ since $\mathcal{V}$ is weakly increasing. Therefore, offer $o^{\prime}$ weakly dominates $o$.

(b) $o \in \mathbb{R}_{+}, \mathcal{V}(\eta+o)-c \leq \mathcal{V}(\eta)$ and $\exists o^{\prime}>o \mathcal{V}(\eta+o)<\mathcal{V}\left(\eta+o^{\prime}\right)$. Then, for all $r<o$, $\Pi_{s}\left(\eta, o^{\prime}, r\right)=\Pi_{s}(\eta, o, r)=0$. For all $r \in\left[o, o^{\prime}\right), \Pi_{s}(\eta, o, r)=\mathcal{V}(\eta+o)-c-\mathcal{V}(\eta) \leq 0=\Pi_{s}\left(\eta, o^{\prime}, r\right)$. And for all $r \geq o^{\prime}, \Pi_{s}(\eta, o, r)=\mathcal{V}(\eta+o)-c-\mathcal{V}(\eta)<\mathcal{V}\left(\eta+o^{\prime}\right)-c-\mathcal{V}(\eta)=\Pi_{s}\left(\eta, o^{\prime}, r\right)$. Therefore, offer $o^{\prime}$ weakly dominates $o$.

(c) $o \in \mathbb{R}_{+}, \exists o^{\prime}<o$ such that $\mathcal{V}\left(\eta+o^{\prime}\right)-c>\mathcal{V}(\eta)$ and $\mathcal{V}\left(\eta+o^{\prime}\right)=\mathcal{V}(\eta+o)$. Then, for all $r<o^{\prime}, \Pi_{s}\left(\eta, o^{\prime}, r\right)=\Pi_{s}(\eta, o, r)=0$. For all $r \in\left[o^{\prime}, o\right), \Pi_{s}\left(\eta, o^{\prime}, r\right)=\mathcal{V}\left(\eta+o^{\prime}\right)-c-\mathcal{V}(\eta)>0=$ 
$\Pi_{s}(\eta, o, r)$. And for all $r \geq o, \Pi_{s}\left(\eta, o^{\prime}, r\right)=\mathcal{V}\left(\eta+o^{\prime}\right)-c-\mathcal{V}(\eta)=\mathcal{V}(\eta+o)-c-\mathcal{V}(\eta)=\Pi_{s}(\eta, o, r)$. Therefore, offer $o^{\prime}$ weakly dominates $o$.

(d) $o=\infty$ and $\exists o^{\prime} \in \mathbb{R}_{+} \mathcal{V}\left(\eta+o^{\prime}\right)-c>\mathcal{V}(\eta)$. Then, for all $r<o^{\prime}, \Pi_{s}\left(\eta, o^{\prime}, r\right)=\Pi_{s}(\eta, o, r)=0$, and for all $r \geq o^{\prime}, \Pi_{s}\left(\eta, o^{\prime}, r\right)=\mathcal{V}\left(\eta+o^{\prime}\right)-c-\mathcal{V}(\eta)>0=\Pi_{s}(\eta, o, r)$. So, offer $o^{\prime}$ weakly dominates $o$.

The results of all four cases contradict the assumption that offer $o$ is weakly undominated. So, $o \in \mathcal{A}(\eta)$. This concludes the proof that $o$ is weakly undominated if and only if $o \in A(\eta)$.

Next, consider the reservation price: $r \in \mathcal{B}(\eta) \Longleftrightarrow r$ is weakly undominated.

“”". Take a reservation price $r \in \mathcal{B}(\eta)$, and suppose that $r$ is weakly dominated by another reservation price $r^{\prime}$. Consider the case $r^{\prime}<r$. For all $o \leq r^{\prime}, \Pi_{b}(\eta, o, r)=u+\mathcal{V}(\eta-o)-\mathcal{V}(\eta)=$ $\Pi_{b}\left(\eta, o, r^{\prime}\right)$. For all $o>r, \Pi_{b}(\eta, o, r)=\Pi_{b}\left(\eta, o, r^{\prime}\right)=0$. That is, reservation prices $r$ and $r^{\prime}$ give the same net payoff for offers on $\left[0, r^{\prime}\right]$ and $(r, \infty)$. Since $r \in \mathcal{B}(\eta)$, two things can happen. In one case, $u+\mathcal{V}(\eta-r) \geq \mathcal{V}(\eta)$. Then, for all $o \in\left(r^{\prime}, r\right], \Pi_{b}(\eta, o, r)=u+\mathcal{V}(\eta-o)-\mathcal{V}(\eta) \geq$ $u+\mathcal{V}(\eta-r)-\mathcal{V}(\eta) \geq 0=\Pi_{b}\left(\eta, o, r^{\prime}\right)$ since $\mathcal{V}$ is weakly increasing. That is, there does not exist an $o \in \mathbb{R}_{+}^{*}$ such that $\Pi_{b}(\eta, o, r)<\Pi_{b}\left(\eta, o, r^{\prime}\right)$. Therefore, $\gamma$ is not weakly dominated by $r^{\prime}$. In another case, there exists an $o \in\left(r^{\prime}, r\right]$ such that $u+\mathcal{V}(\eta-o)>\mathcal{V}(\eta)$. Then, $\Pi_{b}(\eta, o, r)=$ $u+\mathcal{V}(\eta-o)-\mathcal{V}(\eta)>0=\Pi_{b}\left(\eta, o, r^{\prime}\right)$. So, $\gamma$ is also not weakly dominated by $r^{\prime}$.

Consider the case $r<r^{\prime}$. It can be shown as above that reservation prices $r$ and $r^{\prime}$ give the same net payoff for offers on $[0, r]$ and $\left(r^{\prime}, \infty\right)$. For all $o \in\left(r, r^{\prime}\right], u+\mathcal{V}(\eta-o) \leq \mathcal{V}(\eta)$ since $r \in \mathcal{B}(\eta)$. Then, $\Pi_{b}(\eta, o, r)=0 \geq u+\mathcal{V}(\eta-o)-\mathcal{V}(\eta)=\Pi_{b}\left(\eta, o, r^{\prime}\right)$. That is, there does not exist an $o \in \mathbb{R}_{+}^{*}$ such that $\Pi_{b}(\eta, o, r)<\Pi_{b}\left(\eta, o, r^{\prime}\right)$. Therefore, $\gamma$ is not weakly dominated by $r^{\prime}$.

"£". Take a weakly undominated reservation price $r$, and suppose that $r \notin \mathcal{B}(\eta)$. Then, one or both of the following two cases may occur, and I will consider each separately.

(a) $\exists o_{1}>r, u+\mathcal{V}\left(\eta-o_{1}\right)>\mathcal{V}(\eta)$. Compare $o_{1}$ as a reservation price with the given $r$. It is easy to show as above that reservation prices $r$ and $o_{1}$ give the same net payoff for offers on $[0, r]$ and $\left(o_{1}, \infty\right)$. For all $o \in\left(r, o_{1}\right], \mathcal{V}(\eta-o) \geq \mathcal{V}\left(\eta-o_{1}\right)$ since $\mathcal{V}$ is weakly increasing. Then, $\Pi_{b}\left(\eta, o, o_{1}\right)=u+\mathcal{V}(\eta-o)-\mathcal{V}(\eta) \geq u+\mathcal{V}\left(\eta-o_{1}\right)-\mathcal{V}(\eta)>0=\Pi_{b}(\eta, o, r)$. Therefore, reservation price $o_{1}$ weakly dominates $r$.

(b) $u+\mathcal{V}(\eta-r)<\mathcal{V}(\eta)$ and $\exists r^{\prime}<r$ such that $\forall o \in\left(r^{\prime}, r\right), u+\mathcal{V}(\eta-o) \leq \mathcal{V}(\eta)$. Compare $r^{\prime}$ as a reservation price with $r$. It is easy to show that reservation prices $r$ and $r^{\prime}$ give the same net payoff for offers on $\left[0, r^{\prime}\right]$ and $(r, \infty)$. For all $o \in\left(r^{\prime}, r\right), \Pi_{b}(\eta, o, r)=u+\mathcal{V}(\eta-o)-\mathcal{V}(\eta) \leq 0=\Pi_{b}\left(\eta, o, r^{\prime}\right)$, and $\Pi_{b}(\eta, r, r)=u+\mathcal{V}(\eta-r)-\mathcal{V}(\eta)<0=\Pi_{b}\left(\eta, r, r^{\prime}\right)$. Therefore, reservation price $r^{\prime}$ weakly dominates $r$. Since both cases (a) and (b) contradict the assumption that $r$ is weakly undominated, $r \in \mathcal{B}(\eta)$. This concludes the proof.

\section{B. The Proof of Lemma 4}


This lemma states the relationship among $N, m$, and $\frac{M}{p}$, the three variables determining the stationary distribution of money holdings $h$ that satisfies $(17),(19), 0 \leq m \leq 1$, and $0 \leq h(N) \leq$ 1. Note that for any given $N,\left(m, h(N), \frac{M}{p}\right)=(0,1,0)$ and $\left(m, h(N), \frac{M}{p}\right)=\left(\frac{N}{N+1}, \frac{1}{N+1}, \frac{N}{2}\right)$ are solutions to (17) and (19). While the former is a distribution for a nonmonetary equilibrium, the latter corresponds to a uniform distribution of money holdings, that is, $\forall n=0,1, \ldots, N$ $h(n)=\frac{1}{N+1}$. I will focus on other nontrivial stationary distributions for a potential monetary equilibrium. For a given $N$, I will show that $m$ uniquely determines $\frac{M}{p}$ and vice versa.

Take a constant $m$ as given. Rewrite $h(N)$ as $h\left(N ; \frac{M}{p}\right)$ to index the dependence of $h(N)$ on $\frac{M}{p}$ :

$$
h\left(N ; \frac{M}{p}\right)=\frac{m-(1-m) \frac{M}{p}}{m(N+1)-\frac{M}{p}}, \quad 1-h\left(N ; \frac{M}{p}\right)=\frac{m\left(N-\frac{M}{p}\right)}{m(N+1)-\frac{M}{p}} .
$$

Define

$$
T\left(\frac{M}{p}\right) \equiv h\left(N ; \frac{M}{p}\right)\left(1-h\left(N ; \frac{M}{p}\right)\right)^{N}, \quad S(x) \equiv(1-x) x^{N}
$$

$S(m)$ is a positive constant given $m$. Instead of finding solution $\frac{M}{p}$ to (17), I will look for solutions to $T\left(\frac{M}{p}\right)=S(m)$. This can be done in the following two cases, depending on the values of $N$ and $m$.

Case 1. $m(N+1)<N$.

Divide $\mathbb{R}_{+}$into three intervals: $I_{1}=(0, m(N+1)], I_{2}=(m(N+1), N)$, and $I_{3}=[N, \infty)$.

If $\frac{M}{p} \in I_{1}, T\left(\frac{M}{p}\right)$ is decreasing on $I_{1}, T(0)=\frac{N^{N}}{(N+1)^{N+1}}>0$, and $T\left(\frac{M}{p}\right) \rightarrow-\infty$ as $\frac{M}{p} \rightarrow m(N+1)$. Since $S(x)$ is an increasing function when $x<\frac{N}{N+1}$ and by assumption $m<\frac{N}{N+1}, S(m)<S\left(\frac{N}{N+1}\right)=T(0)$. Thus, there exists an $\frac{M}{p}^{*} \in(0, m(N+1))$ such that $T\left(\frac{M}{p}^{*}\right)=S(m)$.

If $\frac{M}{p} \in I_{2}$, by $(B .1), 1-h(N)<0$. There is no solution on $I_{2}$.

If $\frac{M}{p} \in I_{3}, T\left(\frac{M}{p}\right)$ is increasing on $I_{3}, T(N)=0$, and $T\left(\frac{M}{p}\right) \rightarrow S(m)$ as $\frac{M}{p} \rightarrow \infty$. That is, $\forall \frac{M}{p} \in I_{3} T\left(\frac{M}{p}\right)<S(m)$. There is no solution on $I_{3}$.

Case 2. $m(N+1)>N$.

Divide $\mathbb{R}_{+}$into three intervals: $I_{1}=(0, N], I_{2}=(N, m(N+1))$, and $I_{3}=[m(N+1), \infty)$.

If $\frac{M}{p} \in I_{1}, T\left(\frac{M}{p}\right)$ is decreasing on $I_{1}, T(0)=\frac{N^{N}}{(N+1)^{N+1}}>0$, and $T(N)=0<S(m)$.

Since $S(x)$ is a decreasing function when $x>\frac{N}{N+1}$ and by assumption $m \geq \frac{N}{N+1}, S(m)<$ $S\left(\frac{N}{N+1}\right)=T(0)$. Thus, there exists an $\frac{M}{p}^{*} \in(0, N)$ such that $T\left(\frac{M}{p}^{*}\right)=S(m)$.

If $\frac{M}{p} \in I_{2}$, by $(B .1), 1-h(N)<0$. There is no solution on $I_{2}$.

If $\frac{M}{p} \in I_{3}, T\left(\frac{M}{p}\right)$ is increasing on $I_{3}, T\left(\frac{M}{p}\right) \rightarrow-\infty$ as $\frac{M}{p} \rightarrow m(N+1)$, and $T\left(\frac{M}{p}\right) \rightarrow S(m)$ as $\frac{M}{p} \rightarrow \infty$. That is, $\forall \frac{M}{p} \in I_{3} T\left(\frac{M}{p}\right)<S(m)$. There is no solution on $I_{3}$. 
The case of $m(N+1)=N$ corresponds to the special uniform distribution discussed above. To summarize, for a given $N$ and $m$, there exists a unique $\frac{M^{*}}{p}<N$ such that $T\left(\frac{M}{p}^{*}\right)=S(m)$.

Next, I consider the reverse case of finding $m$ for a given $\frac{M}{p}$. From (17) and (19),

$$
\frac{\left(m\left(\frac{M}{p}+1\right)-\frac{M}{p}\right) m^{N}\left(N-\frac{M}{p}\right)^{N}}{\left(m(N+1)-\frac{M}{p}\right)^{N+1}}=(1-m) m^{N} .
$$

Define

$$
O(m) \equiv \frac{(1-m)\left(m(N+1)-\frac{M}{p}\right)^{N+1}}{m\left(\frac{M}{p}+1\right)-\frac{M}{p}}, \quad Q \equiv\left(N-\frac{M}{p}\right)^{N} .
$$

Then (B.3) can be written as

$$
O(m)=Q
$$

Instead of looking for solutions to (17), I will solve for $m$ in (B.5). Given the above result $\left(\left(\frac{M}{p}\right)^{*}<N\right.$ for a given $\left.m\right)$, I need to study only the case of $\frac{M}{p}<N$.

Given that $\frac{M}{p}<N, 0<\frac{M}{p} \frac{1}{N+1}<\frac{M}{p} \frac{1}{\frac{M}{p}+1}<\frac{N}{N+1}$. If $m \in\left(0, \frac{M}{p} \frac{1}{N+1}\right), 1-h(N)<0$. If $m \in\left[\frac{M}{p} \frac{1}{N+1}, \frac{M}{p} \frac{1}{\frac{M}{p}+1}\right), h(N)<0$. So, the only possible solution of $m$ is in $\left[\frac{M}{p} \frac{1}{\frac{M}{p}+1}, 1\right]$. On this interval, $O(m) \rightarrow+\infty$ as $m \rightarrow \frac{M}{p} \frac{1}{\frac{M}{p}+1}$, and $O(1)=0<Q$. Also,

$$
\frac{\partial O}{\partial m}=\frac{\left(m(N+1)-\frac{M}{p}\right)^{N}}{\left(m\left(\frac{M}{p}+1\right)-\frac{M}{p}\right)^{2}}(m(N+1)-N)\left(\frac{M}{p}(N+2)-m(N+1)\left(\frac{M}{p}+1\right)\right) .
$$

The solutions to $\frac{\partial O}{\partial m}=0$ are

$$
m_{1}=\frac{N}{N+1}, \quad m_{2}=\frac{\frac{M}{p}(N+2)}{\left(\frac{M}{p}+1\right)(N+1)}
$$

and $O\left(m_{1}\right)=\left(N-\frac{M}{p}\right)^{N}=Q$. Note that $m_{1}$ gives the special uniform distribution discussed above, which requires that $\frac{M}{p}=\frac{N}{2}$. In this case, $m_{1}=m_{2}$ is the saddle point of $O$, which is the only solution to $(B .5)$. Now consider the cases where $\frac{M}{p} \neq \frac{N}{2}$.

If $\frac{M}{p}<\frac{N}{2}, m_{1}>m_{2}$. Since $O$ is a continuous function of $m$, there exists an $m^{*} \in\left(\frac{M}{p} \frac{1}{\frac{M}{p}+1}, m_{2}\right)$ such that $O\left(m^{*}\right)=\left(N-\frac{M}{p}\right)^{N}=Q$. That is, $m^{*}<m_{2}<m_{1}$, and by $(19), 1-h(N)>m$. Then, by (16), $\{h(n)\}_{n=0}^{N}$ is a decreasing sequence of $n$.

If $\frac{M}{p}>\frac{N}{2}, m_{1}<m_{2}$. Again, since $O$ is a continuous function of $m$, there exists an $m^{*} \in\left(m_{2}, 1\right)$ such that $O\left(m^{*}\right)=\left(N-\frac{M}{p}\right)^{N}=Q$. That is, $m^{*}>m_{2}>m_{1}$, and by $(19), 1-h(N)<m$. Then, by (16), $\{h(n)\}_{n=0}^{N}$ is an increasing sequence of $n$.

This concludes the second part of the proof: for a given $\frac{M}{p}$, there is a unique $m^{*}$ that satisfies $(B .5)$. 


\section{The Derivation of the Value Function}

The solution to the linear difference equation (26) can be represented as a linear combination of the eigenvectors of matrix $\mathcal{T}$. The matrix $\mathcal{T}$ has three distinct eigenvectors, all of which have real eigenvalues. The eigenvalues $\lambda_{0}, \lambda_{1}$, and $\lambda_{2}$ are

$$
\begin{aligned}
& \lambda_{0}=1 \\
& \lambda_{1}=\frac{1}{2}\left(\frac{\phi}{m}+\frac{1-h(N)}{m}+1-\sqrt{\left(\frac{\phi}{m}+\frac{1-h(N)}{m}+1\right)^{2}-\frac{4(1-h(N))}{m}}\right) \in(0,1) \\
& \lambda_{2}=\frac{1}{2}\left(\frac{\phi}{m}+\frac{1-h(N)}{m}+1+\sqrt{\left(\frac{\phi}{m}+\frac{1-h(N)}{m}+1\right)^{2}-\frac{4(1-h(N))}{m}}\right)>1 .
\end{aligned}
$$

The corresponding eigenvectors $\xi_{0}, \xi_{1}$, and $\xi_{2}$ can be expressed as

$$
\xi_{0}=\left(\begin{array}{c}
1 / \phi \\
1 / \phi \\
1
\end{array}\right), \quad \xi_{1}=\left(\begin{array}{c}
\lambda_{1} \\
1 \\
0
\end{array}\right), \quad \xi_{2}=\left(\begin{array}{c}
\lambda_{2} \\
1 \\
0
\end{array}\right)
$$

that satisfy the following relations:

$$
\mathcal{T} \xi_{0}=\xi_{0}, \quad \mathcal{T} \xi_{1}=\lambda_{1} \xi_{1}, \quad \mathcal{T} \xi_{2}=\lambda_{2} \xi_{2}
$$

Define

$$
w^{n+1} \equiv\left(\begin{array}{c}
V(n+1) \\
V(n) \\
\chi
\end{array}\right)
$$

Let $\theta_{0}, \theta_{1}$, and $\theta_{2}$ be the parameters that satisfy

$$
w^{1}=\theta_{0} \xi_{0}+\theta_{1} \xi_{1}+\theta_{2} \xi_{2}
$$

or

$$
\begin{aligned}
\left(\frac{\phi}{m}+1\right) V(0)+c & =\theta_{0} / \phi+\theta_{1} \lambda_{1}+\theta_{2} \lambda_{2} \\
V(0) & =\theta_{0} / \phi+\theta_{1}+\theta_{2} \\
\chi & =\theta_{0}
\end{aligned}
$$

where $(C .6)$ is derived by substituting $V(1)$ solved from (23). By $(C .3)$ and (C.5), induction on $w^{n+1}$ for $n=0,1, \ldots, N-1$ implies that

$$
w^{n+1}=\theta_{0} \xi_{0}+\theta_{1} \lambda_{1}^{n} \xi_{1}+\theta_{2} \lambda_{2}^{n} \xi_{2}=\theta_{0}\left(\begin{array}{c}
1 / \phi \\
1 / \phi \\
1
\end{array}\right)+\theta_{1} \lambda_{1}^{n}\left(\begin{array}{c}
\lambda_{1} \\
1 \\
0
\end{array}\right)+\theta_{2} \lambda_{2}^{n}\left(\begin{array}{c}
\lambda_{2} \\
1 \\
0
\end{array}\right)
$$

Take the second row of $(C .9)$, for $n=1,2, \ldots, N$,

$$
V(n)=\theta_{0} / \phi+\theta_{1} \lambda_{1}^{n}+\theta_{2} \lambda_{2}^{n}
$$


which is the general representation of value function (29). Rewrite equation (25) as

$$
(\phi+1-h(N)) V(N)=(1-h(N))(u+V(N-1))
$$

and substitute (C.10) for $n=N-1$ and $n=N$ into (C.11), I have

$$
(\phi+1-h(N))\left(\theta_{0} / \phi+\theta_{1} \lambda_{1}^{N}+\theta_{2} \lambda_{2}^{N}\right)=(1-h(N))\left(u+\theta_{0} / \phi+\theta_{1} \lambda_{1}^{N-1}+\theta_{2} \lambda_{2}^{N-1}\right) .
$$

Equations $(C .6),(C .7),(C .8)$, and $(C .12)$ are a four-equation linear system with four unknowns $\theta_{0}, \theta_{1}, \theta_{2}$, and $V(0)$. The solution to this linear system is

$$
\begin{aligned}
\theta_{0} & =(1-h(N)) u-m c \\
\theta_{1} & =\frac{1}{G}\left(\left(\lambda_{2}-1-\frac{\phi}{m}\right) m c-\left(\frac{1}{m} \theta_{0}+c\right) F\right) \\
\theta_{2} & =\frac{1}{G}\left(\left(1+\frac{\phi}{m}-\lambda_{1}\right) m c+\left(\frac{1}{m} \theta_{0}+c\right) E\right) \\
V(0) & =\frac{1}{G}\left(\left(\lambda_{2}-\lambda_{1}\right)\left(\theta_{0} F / \phi+m c\right)-\left(c+\left(\lambda_{2}-1\right) \theta_{0} / \phi\right)(F-E)\right)
\end{aligned}
$$

where

$$
\begin{aligned}
& E=\phi \lambda_{1}^{N}-\lambda_{1}^{N-1}(1-h(N))\left(1-\lambda_{1}\right) \\
& F=\phi \lambda_{2}^{N}+\lambda_{2}^{N-1}(1-h(N))\left(\lambda_{2}-1\right) \\
& G=\left(1-\lambda_{1}+\frac{\phi}{m}\right) F+\left(\lambda_{2}-1-\frac{\phi}{m}\right) E
\end{aligned}
$$

Equations $(C .10)$ and (C.13) fully characterize the value function in terms of the parameters of the model $u, c$, and $\phi$; the upper bound on money holdings $N$; and the distribution parameter $m$ and $h(N)$.

\section{The Proof of Lemma 7}

The concavity of the value function in $n$ for $N \leq n \leq K$ can be directly shown by equations (25) and (29); that is, for $n=N, \ldots, K-1$,

$$
V(n)-V(n-1)>V(n+1)-V(n) .
$$

I will focus on the case where $n<N$. By equations (24) and (25),

$$
\begin{aligned}
& (1-h(N))((V(N-1)-V(N-2))-(V(N)-V(N-1)))= \\
& \phi(V(N)-V(N-1))+m(V(N)-c-V(N-1)) .
\end{aligned}
$$

The right side of $(D .2)$ is positive, since $V(N)>V(N-1)$ by Lemma 5 , and $V(N)-c>V(N-1)$ by (36). Therefore,

$$
V(N-1)-V(N-2)>V(N)-V(N-1)
$$

Similarly, subtract equation (24) for $n$ from that of $n-1$ and rearrange the difference, I have 


$$
\begin{array}{r}
(1-h(N))((V(n-1)-V(n-2))-(V(n)-V(n-1)))=\phi(V(n)-V(n-1)) \\
+m((V(n)-V(n-1))-(V(n+1)-v(n))) .
\end{array}
$$

Again, the right side of (D.4) is positive for $n=N-1$ by Lemma 5 and by inequality (D.3). So, $V(N-2)-V(N-3)>V(N-1)-V(N-2)$. Backward induction on $n$ using (D.4) yields that for $n=1,2, \ldots, N-1$,

$$
V(n)-V(n-1)>V(n+1)-V(n) .
$$

Inequalities (D.1) and (D.5) give the statement of Lemma 7.

\section{E. The Proof of Lemma 12}

To show that $\forall n>K, \rho(n p) \geq k p$, it is sufficient to show that $\forall n>K, V(n)-V(n-J) \leq u$. By the definition of $K$ in $(38), V(K+1)-V(K+1-J) \leq u$. Consider the condition for $n$ in each of the following three cases in sequence.

Case 1. $K+2 \leq n \leq N-1+J$ (or $K-J+2 \leq n-J \leq N-1)$. By equations (24) and (30),

$$
\begin{aligned}
(\phi+1)(V(n)-V(n-J))= & (1-h(N))(V(n-1)-V(n-J-1))+h(N) u \\
& -m(V(n-J+1)-c-V(n-J)) \\
\leq & (1-h(N))(V(n-1)-V(n-J-1))+h(N) u
\end{aligned}
$$

since $V(n-J+1)-c<V(n-J)$ by the concavity of the value function and (36). Given $V(K+1)-V(K+1-J) \leq u$, starting with $n=K+2$ and applying (E.1) recursively, I get $V(n)-V(n-J) \leq u$.

Case 2. $N+J \leq n \leq K+J$ (or $N \leq n-J \leq K)$. By equations (29) and (30),

$$
(\phi+1)(V(n)-V(n-J))=(1-h(N))(V(n-1)-V(n-J-1))+h(N) u .
$$

Using the results of Case 1 and applying (E.2) recursively starting from $n=N+J$, I get $V(n)-V(n-J) \leq u$.

Case 3. $n \geq K+J+1$ (or $n-J \geq K+1)$. By equation (30),

$$
\begin{gathered}
(\phi+1)(V(n)-V(n-J))=(1-h(N))(V(n-1)-V(n-J-1)) \\
+h(N)(V(n-J)-V(n-2 J)) .
\end{gathered}
$$


Using the results of previous cases and applying (E.3) recursively starting from $n=K+J+1$, I get $V(n)-V(n-J) \leq u$.

\section{F. The Proof of Lemma 14}

Consider an agent with money holding $n p, n \geq N$. To show $\omega(n p)>p$, it is sufficient to show that $p \notin \mathcal{A}(n p)$, or equivalently, $V(n+1)-V(n) \leq c$.

Consider the case that $n \in[N, N+\bar{l}-1)$, where $\bar{l}=\max \left\{K+1-N, J^{*}\right\}$. By the definition of $J^{*}$, $V\left(N+J^{*}-1\right)-V(N) \leq c$. Since $V$ is increasing, for any $n \in\left[N, N+J^{*}-1\right), V(n+1)-V(n) \leq c$. Also by the concavity of the value function and by (37), for any $n \in[N, K), V(n+1)-V(n) \leq$ $V(N+1)-V(N) \leq c$. That is, for any $n \in[N, N+\bar{l}-1), V(n+1)-V(n) \leq c$.

If $\bar{l}>J$, or equivalently, $K+1-J>N$, condition (43) is vacuous. I need only to consider the case where $n \geq N+\bar{l}-1=K$. By the definition of $K, V(K+1)-V(K+1-J) \leq u<V(K)-V(K-J)$, or $V(K+1)-V(K) \leq V(K+1-J))-V(K-J) \leq c$ since $K+1-J>N$ and by the result above for $n \in[N, N+\bar{l}-1)$. For $n \geq K+1$, by equation (30),

$$
\begin{aligned}
(\phi+1)(V(n+1)-V(n))=(1-h(N))(V(n)-V(n-1))+ & \\
& h(N)(V(n-1)-V(n-2)) .
\end{aligned}
$$

Applying (F.1) recursively, given $V(K+1)-V(K) \leq c$, I get $V(n+1)-V(n) \leq c$ for all $n \geq K+1$.

Consider the case where $\bar{l} \leq J$, or equivalently, $K+1 \leq N+J$. By (43), for $n \in[N+\bar{l}-1, N+J)$, $V(n+1)-V(n) \leq c$. For $n \geq N+J$, applying (F.1) recursively, given $V(N+J)-V(N+J-1) \leq c$, I get $V(n+1)-V(n) \leq c$.

Combining all above cases, for all $n \geq N$, I conclude that $V(n+1)-V(n) \leq c$. 


\section{REFERENCES}

1. G. Camera and D. Corbae, "Monetary Patterns of Exchange with Search," manuscript, Department of Economics, University of Iowa, 1996.

2. D. Gale, "Money, in Equilibrium," Cambridge University Press, New York, 1982.

3. E. J. Green and R. Zhou, A rudimentary model of search with divisible money and prices, J. Econ. Theory (forthcoming).

4. N. Kiyotaki and R. Wright, A contribution to the pure theory of money, J. Econ. Theory 53 (1991), 215-235.

5. N. Kiyotaki and R. Wright, A search-theoretic approach to monetary economics, Amer. Econ. Rev. 83 (1993), 63-77.

6. M. Molico, "The Distribution of Money and Prices in a Search Equilibrium," manuscript, Department of Economics, University of Pennsylvania, 1996.

7. R. B. Myerson, "Game Theory: Analysis of Conflict," Harvard University Press, Cambridge, Mass., 1991.

8. G. Rocheteau, "Is There a Real-Balance Effect in the Search-Theoretic Model of Fiat Money?" manuscript, CEFIB, Université Panthéon-Assas, 1997.

9. S. Shi, Money and prices: A model of search and bargaining, J. Econ. Theory 67 (1995), 467-496.

10. A. Trejos and R. Wright, Search, bargaining, money, and prices, J. Polit. Econ. 103 (1995), $118-141$.

11. N. Wallace, "Questions Concerning Rate-of-Return Dominance and Indeterminacy in Absenceof-Double-Coincidence Models of Money," manuscript, Department of Economics, University of Miami, 1996. 